\title{
EchoGéo
}

$54 \mid 2020$

Varia

\section{Géographie des élections européennes de 2019}

Réalignement des électorats et renouvellement des oppositions entre centre et périphérie dans un contexte de crise systémique

Christian Vandermotten, Pablo Medina Lockhart et Herman van der Wusten

\section{OpenEdition}

\section{Journals}

Édition électronique

URL : https://journals.openedition.org/echogeo/20473

DOI : $10.4000 /$ echogeo.20473

ISSN : 1963-1197

Éditeur

Pôle de recherche pour l'organisation et la diffusion de l'information géographique (CNRS UMR 8586)

Référence électronique

Christian Vandermotten, Pablo Medina Lockhart et Herman van der Wusten, « Géographie des élections européennes de 2019 », EchoGéo [En ligne], 54 | 2020, mis en ligne le 01 février 2021, consulté le 26 août 2021. URL : http://journals.openedition.org/echogeo/20473 ; DOI : https://doi.org/ $10.4000 /$ echogeo. 20473

Ce document a été généré automatiquement le 26 août 2021.

EchoGéo est mis à disposition selon les termes de la licence Creative Commons Attribution - Pas d'Utilisation Commerciale - Pas de Modification 4.0 International (CC BY-NC-ND) 


\section{Géographie des élections européennes de 2019}

Réalignement des électorats et renouvellement des oppositions entre centre et périphérie dans un contexte de crise systémique

\section{Christian Vandermotten, Pablo Medina Lockhart et Herman van der Wusten}

\section{Introduction}

1 Les élections européennes offrent un panorama global et synchrone de l'opinion publique européenne. Néanmoins, elles ne se déroulent pas dans les mêmes conjonctures politiques nationales. Certains électeurs les considèrent comme de second ordre : les taux d'abstention y sont en général plus élevés qu'aux élections nationales.

2 Leur analyse d'ensemble repose presque toujours sur celle d'une somme de scrutins nationaux ou sur des sondages des motivations des électeurs (Reif \& Schmitt, 1980 ; Van der Eijk et Franklin, 1996; Perrineau, 2005; EVS, 2008; Hix et Marsh, 2011). Ces analyses ont été encadrées par des mises à jour de la théorie des clivages et des interrogations sur les évolutions des articulations entre classes sociales et comportements électoraux (Franklin et al., 1992 ; Dogan, 1996; Evans, 1999; Ignazi, 1999 ; Clark et Lipset, 2001 ; Gordon, 2018 ; Hooghe et Marks, 2018).

3 Si le niveau national reste un contexte déterminant et indispensable pour l'interprétation des résultats, il convient cependant de situer ceux-ci et leurs évolutions dans un cadre systémique plus large. À l'opposé, un regard infranational permet de mieux appréhender les impacts de ces contextes systémiques globaux à travers les différenciations spatiales des réponses politiques qu'ils suscitent. Ceci en éclaire les fondements.

4 Pourtant, les analyses infranationales menées à l'échelle de l'ensemble des pays membres de l'Union européenne sont assez rares. Un travail pionnier a été l'Atlas électoral européen de Vanlaer (1984), suivi par plusieurs publications réalisées au 
Laboratoire de géographie de l'Université libre de Bruxelles (e.a. Vandermotten, Van Hamme, Medina Lockhart). Citons aussi Dijkstra, Poelman et Rodriguez-Pose (2019).

5 L'objectif de cet article est d'examiner l'articulation entre cadres systémiques globaux, résultats électoraux nationaux et configurations infranationales aux élections européennes de 2019, en la resituant dans une perspective temporelle. Il s'agit d'examiner la géographie infranationale des résultats, au-delà des scores partisans nationaux, pour mettre en évidence des déterminants structuraux communs aux divers pays européens. Une analyse à l'échelle européenne impose tout d'abord un classement des partis en familles pertinentes (Van der Eijk et Franklin, 1996; Vandermotten et Vandeburie, 2011).

\section{Les familles politiques}

6 Les alliances politiques et les groupes parlementaires européens fournissent une indication de base pour classer les partis politiques. Toutefois, cela n'est pas suffisant : certains groupes sont hétérogènes. Ils rassemblent des partis très différents les uns des autres, tant quant à leur positionnement sur l'axe gauche-droite que sur d'autres enjeux majeurs, dont l'euroscepticisme. Certains partis ont changé de groupe politique au cours du temps. Parmi les petits partis non représentés au Parlement européen, certains font partie d'alliances politiques européennes, mais d'autres pas.

7 Pour obtenir l'analyse la plus robuste possible, le spectre politique a été subdivisé en sept familles :

- l'extrême-gauche, y compris les partis eurosceptiques ou populistes qui relèvent de cette orientation et les alliances entre extrême-gauche et écologistes radicaux ;

- la gauche de tradition sociale-démocrate ;

- les écologistes, y compris les partis visant la protection animale et les partis pirates ;

- les libéraux ;

- les démocrates-chrétiens ou conservateurs europhiles;

- la droite affirmée, conservateurs identitaires et/ou eurosceptiques à des degrés divers, mais néanmoins bien enracinés, voire dominants, dans leurs establishments politiques nationaux (conservateurs britanniques, N-VA flamande, PiS polonais, FIDESz hongrois, etc.);

- la droite extrême, très eurosceptique, très anti-immigrés, populiste ou plus ou moins fascisante, plus ou moins radicalement ultra-conservatrice sur le plan sociétal.

8 En outre, une catégorie "divers", peu fournie, comprend des partis inclassables, mineurs, catégoriels, ou des candidats isolés ou peu connus.

Le rattachement des partis à ces familles a été fondé sur différents critères :

- leur appartenance à un groupe au Parlement européen. Les familles ci-dessus correspondent respectivement, mais avec des exceptions importantes, aux groupes GUE/NGL, S\&D, Verts/ALE, Renew (ex-ALDE), PPE, CRE et ID (ex-ELDD et ENL). La majorité des non-inscrits relève aussi de la droite extrême ;

- leur appartenance à une alliance européenne ou à une internationale partisane ;

- leur positionnement programmatique, en particulier sur un axe gauche-droite ou par rapport à la construction européenne. Parfois, ceci a pu l'emporter dans le choix classificatoire sur l'appartenance au groupe politique (par exemple pour le FIDESz hongrois ou le KDH slovaque). 

programmatiques d'un parti peuvent varier au cours du temps: on assiste ainsi globalement depuis une cinquantaine d'années à une droitisation du positionnement des partis de la famille sociale-démocrate. En outre, certains partis connaissent des tensions internes entre europhiles et eurosceptiques ou europhobes, comme parmi des partis de droite classique tiraillés entre support au néo-libéralisme et à l'économie mondialisée et nationalisme conservateur. Elles ont atteint leur paroxysme chez les conservateurs britanniques (Hooghe et Marks, 2018). Enfin, les attitudes des électeurs peuvent ne pas être entièrement similaires à celles des directions des partis pour lesquels ils votent. L'électeur moyen se positionne en général plus à droite sur l'axe des valeurs que les directions des partis, sauf les électeurs des partis de droite extrême (Trechsel et Mair, 2009 ; Vandermotten et Vandeburie, 2011).

12 Outre les scores obtenus par les diverses familles politiques, il convient aussi d'intégrer à l'analyse le taux de participation au scrutin ${ }^{1}$.

\section{Les cartes infranationales}

13 Les résultats des familles politiques aux élections de 2019 sont cartographiés à un niveau de désagrégation le plus homogène possible. Il combine les unités de niveau NUTS 3 ou NUTS 2 selon les pays (illustrations 1 à 9) ${ }^{2}$. Ceci permet d'examiner les comportements électoraux régionaux à une échelle où des séries suffisamment étoffées de données contextuelles sont disponibles auprès d'EUROSTAT. Une désagrégation plus poussée, pour laquelle les données ne seraient pas disponibles pour une série de pays, pourrait révéler d'autres dimensions explicatives aux comportements électoraux, comme des différences entre les centres-villes et leurs espaces périurbains ( $c f$. pour la France, Lévy, 2013, 2017). 
Illustration 1 - Votes exprimés en faveur de l'extrême-gauche (partis principalement rattachés au groupe GUE/GVN) (en\%)

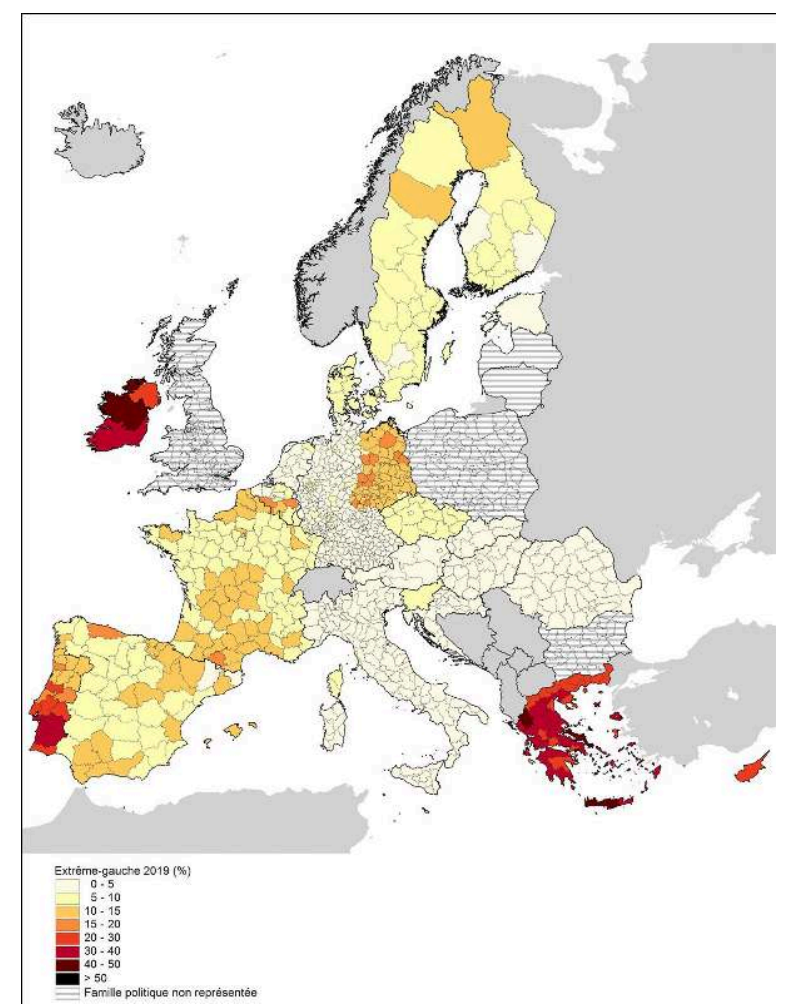

Illustration 2 - Votes exprimés en faveur de la social-démocratie (partis principalement rattachés au groupe S\&D) (en \%)

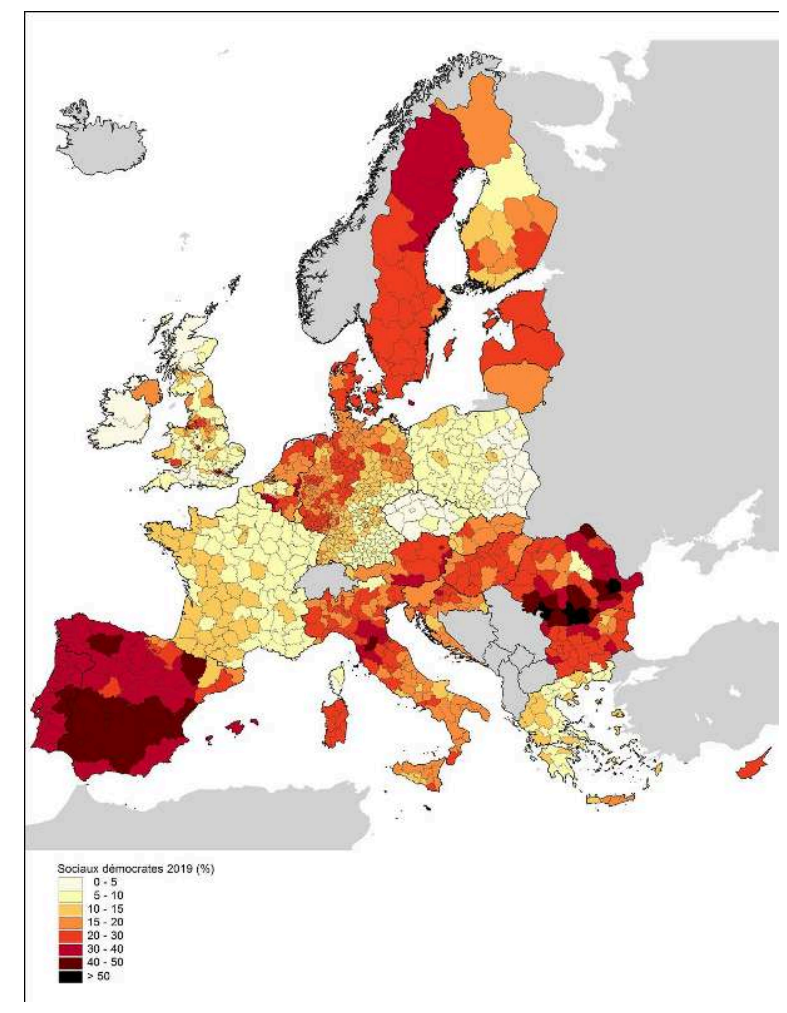


Illustration 3 - Votes exprimés en faveur des écologistes (partis principalement rattachés au groupe Verts/ALE) (en \%)

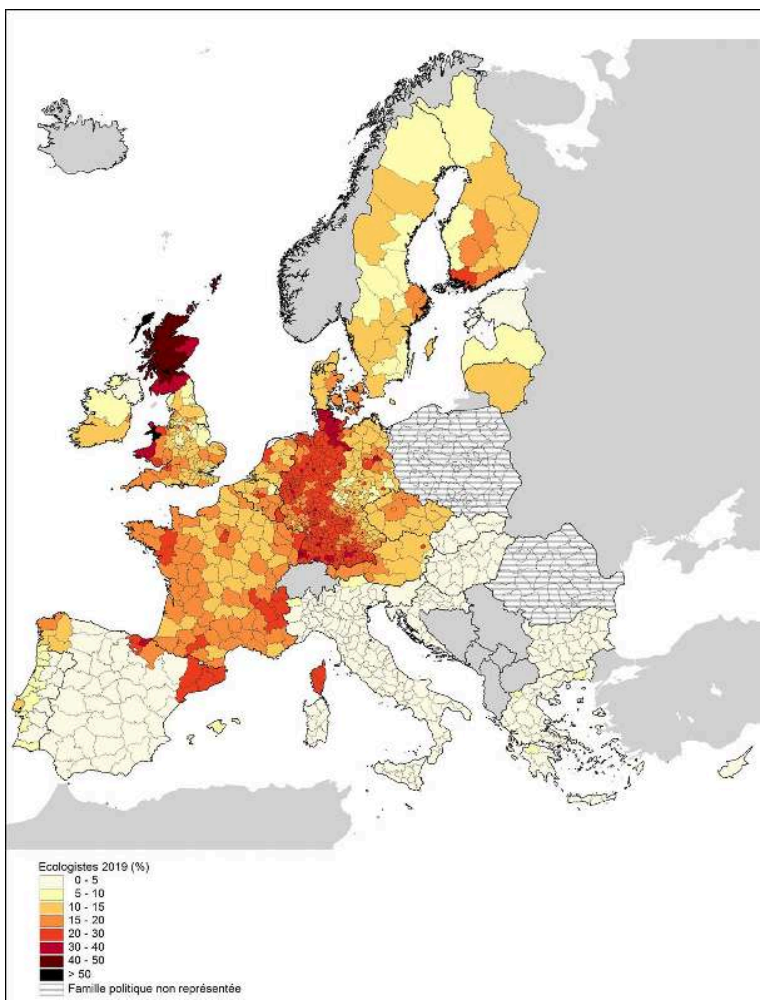

Illustration 4 - Votes exprimés en faveur des libéraux (partis principalement rattachés au groupe Renew, ex-ALDE) (en\%)

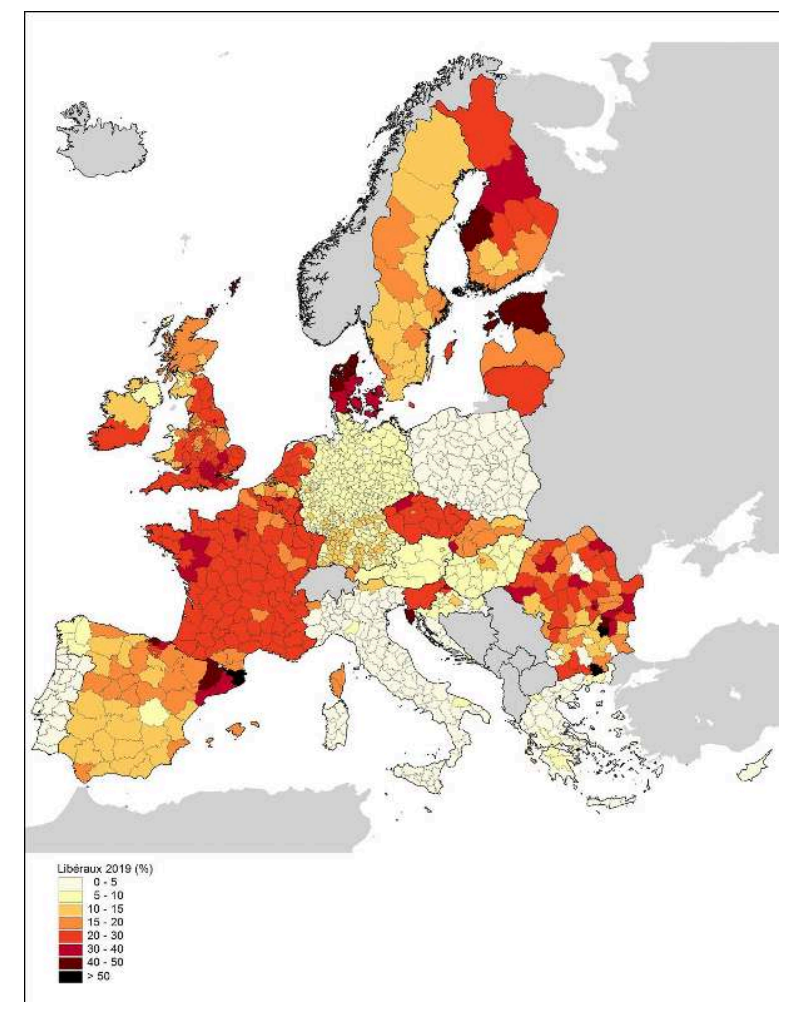


Illustration 5 - Votes exprimés en faveur de la démocratie-chrétienne et des conservateurs europhiles (partis principalement rattachés au groupe PPE) (en \%)

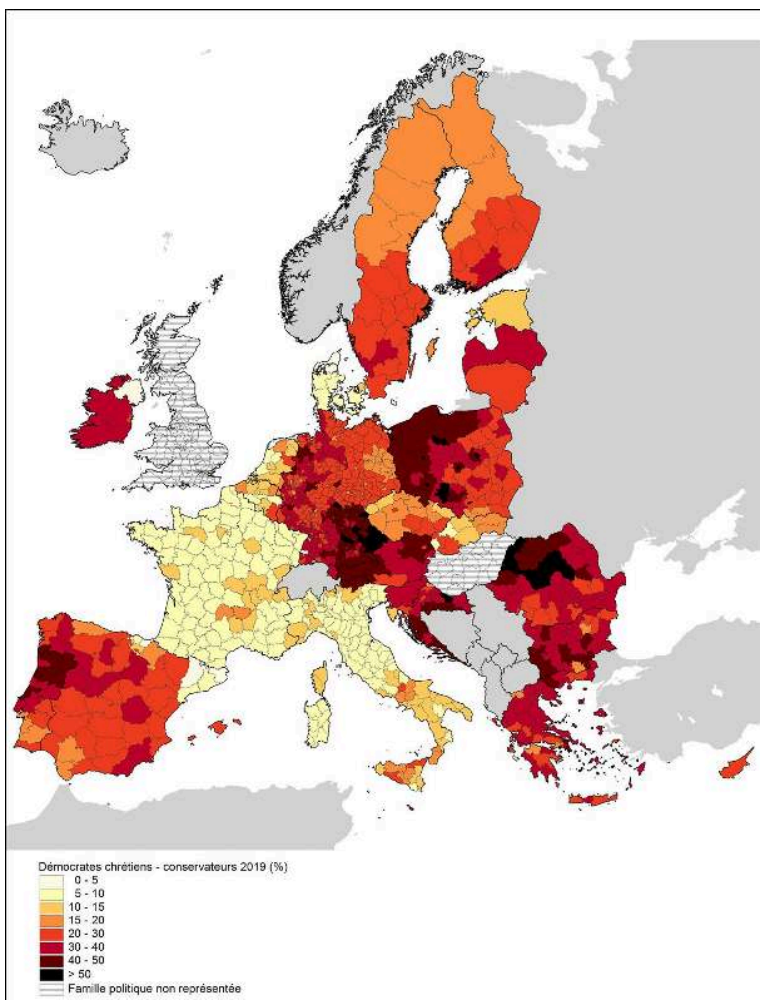

Illustration 6 - Votes exprimés en faveur des conservateurs eurosceptiques (partis principalement rattachés au groupe $\mathrm{CRE}$ ) (en \%)

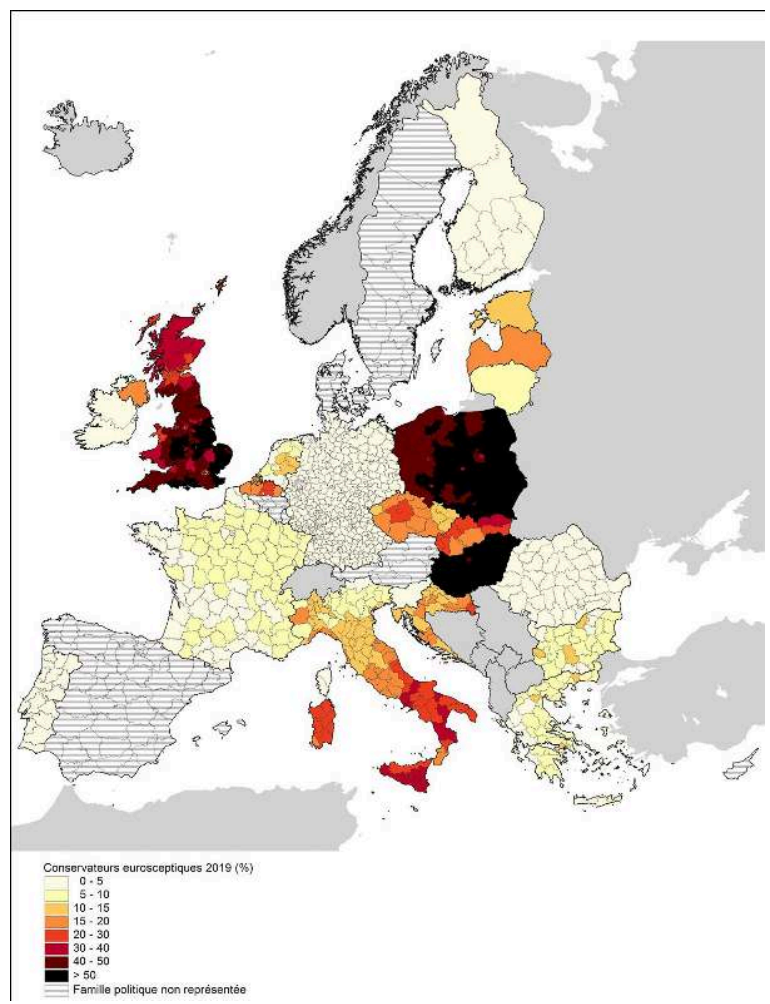


Illustration 7 - Votes exprimés en faveur de la droite extrême (partis principalement rattachés au groupe ID, ex-ENL, ou non-inscrits) (en \%)

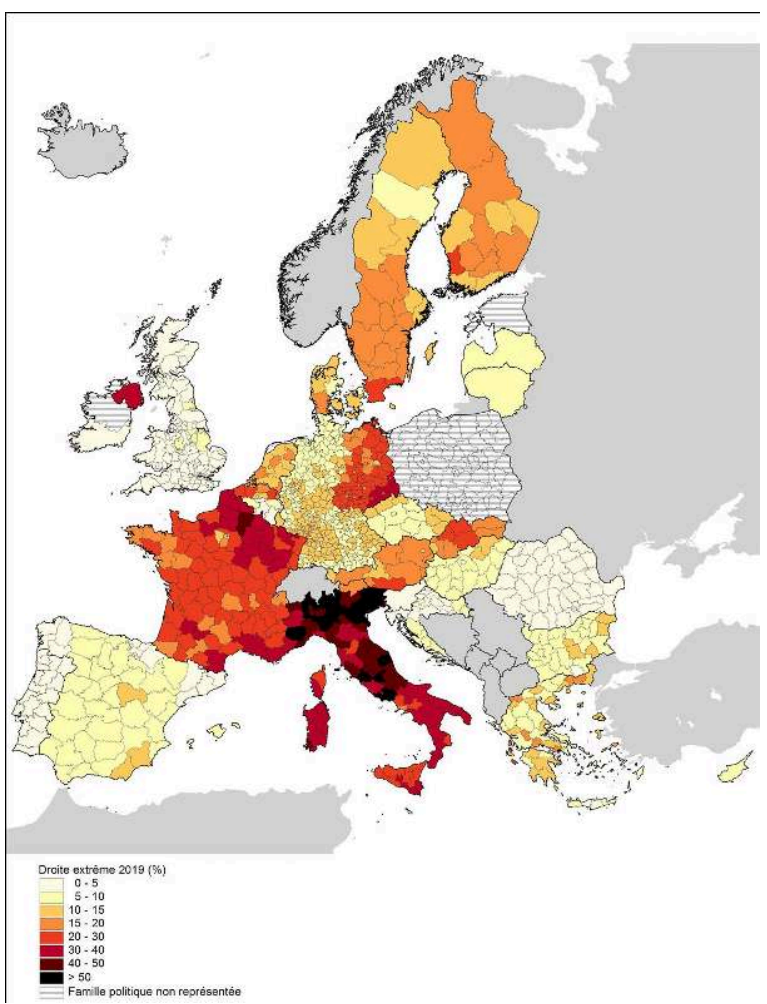

Illustration 8 - Votes exprimés en faveur des conservateurs eurosceptiques et de la droite extrême (en \%)

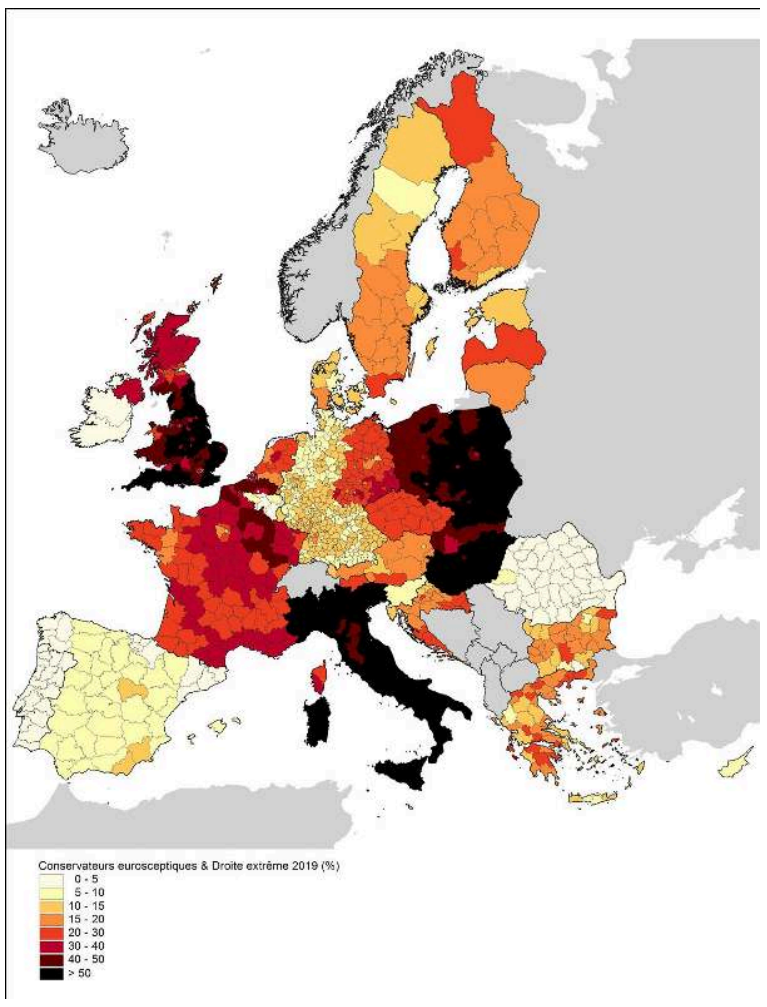




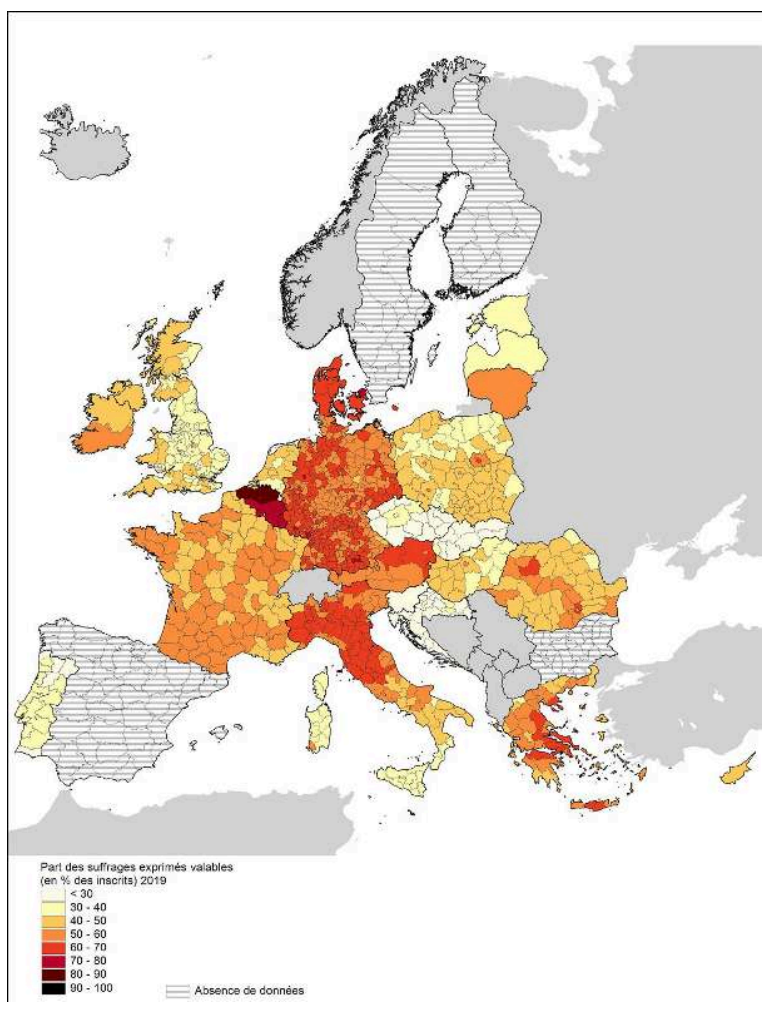

\section{La surdétermination des cadres étatiques}

Les cartes montrent d'emblée le poids des cadres nationaux, tant pour les résultats que pour la participation au vote. Les élections européennes restent donc avant tout l'addition de 28 scrutins nationaux. Selon les familles politiques, entre $67 \%$ et $88 \%$ de la variance des résultats, au niveau de désagrégation géographique utilisé ici, est imputable aux cadres étatiques (tableau 1). Cette importance des déterminants nationaux est la plus nette pour les résultats des partis situés aux extrêmes de l'échiquier politique, un peu moins pour les écologistes et pour les partis de l'establishment.

Tableau 1 - Variance totale et variance inter-États des résultats des familles politiques (les données sont pondérées par le nombre de suffrages exprimés)

\begin{tabular}{|c|c|c|c|c|c|c|c|c|}
\hline & $\begin{array}{c}\text { Extrême- } \\
\text { gauche }\end{array}$ & $\begin{array}{c}\text { Sociaux- } \\
\text { démocrates }\end{array}$ & Ecologistes & Libéraux & $\begin{array}{c}\text { Dém.- } \\
\text { chrétiens, } \\
\text { droite } \\
\text { classique }\end{array}$ & $\begin{array}{c}\text { Cons. } \\
\text { Euroscept. }\end{array}$ & $\begin{array}{c}\text { Droite } \\
\text { extrême }\end{array}$ & Divers \\
\hline Pourcentage & 6,4 & 18,9 & 12,0 & 14,2 & 19,9 & 12,7 & 14,5 & 1,5 \\
\hline Variance totale & 58,7 & 112,2 & 106,9 & 119,2 & 198,6 & 332,4 & 195,8 & 4,0 \\
\hline $\begin{array}{c}\text { Variance inter- } \\
\text { Etats }\end{array}$ & 49,8 & 75,6 & 73,4 & 89,1 & 151,1 & 291,0 & 161,0 & 3,3 \\
\hline $\begin{array}{c}\text { Part de la } \\
\text { variance inter- } \\
\text { Etats dans la } \\
\text { variance totale }\end{array}$ & 0,85 & 0,67 & 0,69 & 0,75 & 0,76 & 0,88 & 0,82 & 0,83 \\
\hline
\end{tabular}

Pour rappel, dans une population statistique divisée en sous-populations, la variance totale est égale à la somme de la variance entre les sous-populations et des variances à l'intérieur des souspopulations. 

des États-nations et dans l'histoire, sur les temps longs, des spécificités de leurs rapports de classes, de leurs variétés linguistiques et religieuses, des rapports entre centre et périphérie, de la mise en place de leurs systèmes partisans, qui se sont développés sur la base de coalitions en compétition pour la participation ou le contrôle du pouvoir central (Flora, 1999). Ces anciens clivages semblent parfois dépassés, mais ils continuent néanmoins à modeler bien des aspects de la vie sociale. Divers modèles de l'État social se sont développés en Europe. Leurs bases subsistent pour l'essentiel, même s'ils ont été partiellement démantelés ces dernières décennies. Ils couvrent l'assistance, la régulation du travail, les retraites, mais aussi l'éducation, le logement. Ils ont généré d'importantes différences entre les sociétés nationales. Elles ont conditionné et se transcrivent dans les préférences électorales (Hemerijck, 2013).

L'intégration des communautés locales en États-nations homogènes a été réalisée avec plus ou moins de succès (Deutsch et Foltz, 1966; Gellner, 1983). Là où elle fut la moins achevée, on peut observer des tentatives d'affirmation de nouvelles nations. La construction européenne et la mondialisation rendent le rôle et la légitimité de l'Étatnation moins évidents. En effet retour, on observe un repli sur le local et les identités communautaires. On peut ainsi constater des frontières internes sur les cartes électorales en Espagne, en Belgique, au Royaume-Uni. En Allemagne, une frontièrefantôme se dessine entre les ex-RFA et RDA, même si ici les écarts socio-économiques rendent compte à eux seuls des différences de comportements électoraux. Dans d'autres pays encore la carte électorale révèle une réalisation imparfaite de l'homogénéisation nationale, comme en Italie.

17 Au-delà du prescrit du recours à la représentation proportionnelle aux élections européennes, les modalités de cette consultation diffèrent sensiblement d'un pays à l'autre. Dans quelques pays, le vote est théoriquement obligatoire (Belgique, Luxembourg). Des pays sont divisés en subdivisions électorales, dans d'autres prévaut une circonscription nationale unique. Certains déterminent des seuils électoraux de représentation des partis, d'autres pas. Tout cela peut soit faciliter, soit compliquer l'émergence de nouveaux partis et influencer les inerties du système partisan. Il convient aussi de se rappeler des fortes variations du nombre de voix nécessaires pour décrocher un siège: un parlementaire européen luxembourgeois est élu avec en moyenne 34000 suffrages exprimés, un Français avec 302 000, un Belge avec 317000. Ces facteurs organisationnels renforcent la tendance à transformer l'élection européenne en une addition de scrutins nationaux (Van der Eijk et Franklin, 1996).

\section{Un contexte de crise systémique}

18 Le contexte socio-économique global et son impact dans les différents États doit aussi être considéré pour comprendre les choix politiques en 2019 et leurs évolutions antérieures.

19 Une crise systémique longue et globale a débuté au milieu des années 1970 avec l'essoufflement du modèle fordiste keynésien. Le ralentissement tendanciel de la croissance économique qui en a résulté est particulièrement sensible en Europe, plus qu'aux États-Unis. À l'épuisement du modèle fordiste, les détenteurs du capital ont répondu par des glissements massifs de la production industrielle vers des pays à bas 
salaires. Aujourd'hui, certains de ces pays, au premier rang desquels la Chine, ont affirmé leur place dans le système économique mondialisé et maîtrisent aussi les segments de haute technologie.

Mus par l'essor de l'idéologie néo-libérale et monétariste, les États ont mis en place à partir des années 1970, avec plus ou moins de vigueur en fonction de leurs situations objectives et des rapports de force politiques existants, des politiques d'austérité, de dérégulation et d'affaiblissement des transferts sociaux.

Des phases d'atonie de l'investissement, tant public que privé, ont entraîné une croissance médiocre de la productivité, malgré une révolution technologique. Combinée aux déplacements de larges segments de la production industrielle vers des pays à bas salaires, celle-ci a détruit du travail non qualifié. La classe ouvrière traditionnelle, sa capacité de résistance syndicale, ses structures d'encadrement en ont été drastiquement affaiblies. On observe aussi un déclassement et une prolétarisation des classes moyennes inférieures (indépendants, employés et cadres intermédiaires, par ex. dans le secteur bancaire), le détricotage des statuts des fonctionnaires, une concurrence des migrants sur le travail peu qualifié. La part du travail s'est réduite au profit de celle du capital dans la répartition du revenu (réduction qui se révélerait plus forte encore si on ajoutait aux revenus du capital les rémunérations extravagantes du haut management). Les inégalités sociales s'accroissent, même si elles restent plus faibles qu'aux États-Unis (et qu'en Chine « communiste »!) (tableau 2).

Tableau 2 - Évolution des inégalités sociales en Europe

\begin{tabular}{|l|c|c|c|c|c|c|c|c|}
\hline \multirow{2}{*}{} & \multicolumn{4}{|c|}{ Indice de Gini } & \multicolumn{3}{c|}{ Part des revenus détenue par les 20\% } \\
\cline { 2 - 9 } & \multicolumn{1}{|c|}{1980 's } & 1990 's & 2000 's & 2010 's & 1980 's & 1990 's & 2000 's & 2010 's \\
\hline $\begin{array}{l}\text { Europe du nord-ouest } \\
\text { (sans le Royaume-Uni) }\end{array}$ & $\ldots$ & $\ldots$ & 30,7 & 31,2 & $\ldots$ & $\ldots$ & 8,4 & 8,2 \\
\hline Royaume-Uni & $\ldots$ & $\ldots$ & 34,8 & 33,4 & $\ldots$ & $\ldots$ & 7,0 & 7,4 \\
\hline Europe nordique & $\ldots$ & $\ldots$ & 26,7 & 27,9 & $\ldots$ & $\ldots$ & 9,3 & 8,9 \\
\hline Europe méditerranéenne & $\ldots$ & $\ldots$ & 34,0 & 35,4 & $\ldots$ & $\ldots$ & 6,8 & 6,2 \\
\hline Europe centre-orientale & 24,5 & 28,1 & 33,4 & 32,6 & 9,9 & 9,1 & 7,1 & 7,1 \\
\hline États-Unis & 37,5 & 39,7 & 40,7 & 41,0 & 5,5 & 5,4 & 5,2 & 5,1 \\
\hline Chine & $\ldots$ & 35,4 & 42,0 & 41,0 & $\ldots$ & 7,4 & 5,5 & 5,8 \\
\hline
\end{tabular}

Source : Banque mondiale.

La financiarisation spéculative de l'économie a entraîné l'apparition de bulles. La crise de 2008 a imposé aux États d'intervenir massivement pour sauver les banques, entraînant de nouvelles mesures d'austérité. Sur le plan écologique, la mondialisation et la dérégulation ont accentué les charges environnementales (détournement des normes, transports à longue distance, transports routiers, emballages, etc.). En même temps, la faiblesse des investissements, en particulier publics, retarde l'essor de technologies plus propres, le développement des transports en commun, etc. Dès lors, la problématique du changement climatique et l'écologie politique remettent en question l'économie consumériste, voire les logiques du système capitaliste globalisé. Elles s'imposent à l'agenda politique dans les dernières années. 


\section{La déclinaison de la crise à travers l'espace européen ${ }^{3}$}

La mondialisation favorise les zones les mieux insérées dans les réseaux internationaux, les grandes aires métropolitaines de l'Europe du nord-ouest, aux dépens des zones périphériques et des régions de vieille industrialisation. En même temps, le glissement d'objectifs privilégiant la recherche de l'équité vers celle de la compétitivité a entraîné une mutation des politiques régionales européennes. L'aide aux régions défavorisées tend à se réduire au profit des zones les plus "efficaces", mises en concurrence entre elles.

Au cœur de l'Europe, l'Allemagne, qui bénéficie de son avance technologique, entend maintenir un euro fort, qui impose des politiques d'austérité à l'ensemble de l'Union. Elles sont plus marquées qu'aux États-Unis, où les dépenses sociales pèsent moins dans le budget et qui n'hésitent pas à profiter du statut de monnaie de réserve mondiale du dollar pour financer un large déficit budgétaire. Alors que l'Allemagne se fonde sur ses avantages compétitifs industriels, le Royaume-Uni, dont l'économie est très financiarisée et très mondialisée, vise une dérégulation maximale : il ne s'accommode plus d'une Europe allant au-delà d'un grand marché et aboutit au Brexit.

Tableau 3 - Quelques indicateurs macroéconomiques et démographiques dans les différentes parties de l'Union européenne

\begin{tabular}{|c|c|c|c|c|c|c|c|c|}
\hline & \multirow{2}{*}{\begin{tabular}{|c|}
$\begin{array}{c}\text { Indice de } \\
\text { contrôle de la } \\
\text { corruption }\end{array}$ \\
\\
2018 \\
\end{tabular}} & \multirow{2}{*}{\begin{tabular}{|c|} 
\% de la \\
logistique, \\
des services \\
financiers \\
(sauf \\
l'immobilier) \\
et des \\
services aux \\
entreprises \\
dans le PIB \\
\\
2017 \\
\end{tabular}} & \multirow{2}{*}{$\begin{array}{l}\begin{array}{r}\text { \% de la R-D } \\
\text { dans le PIB }\end{array} \\
2018 \\
\end{array}$} & \multirow{2}{*}{$\begin{array}{c}\text { \% de la main- } \\
\text { d'oesurre de } \\
15-64 \text { ans } \\
\text { possédart un } \\
\text { diplome } \\
\text { supérieur } \\
\text { et/ou } \\
\text { travillant } \\
\text { dans le } \\
\text { domaine des } \\
\text { sciences et } \\
\text { technologies } \\
2018 \\
2018\end{array}$} & \multirow{2}{*}{$\begin{array}{c}\text { Evolution de } \\
\text { la population } \\
1990-2018 \\
\\
1990-2018\end{array}$} & \multicolumn{3}{|c|}{$\begin{array}{c}\text { PIB/hab. (Europe du Nord-Ouest = } \\
100)\end{array}$} \\
\hline & & & & & & 1995 & 2010 & 2018 \\
\hline $\begin{array}{l}\text { Europe du nord- } \\
\text { ouest }\end{array}$ & 1,7 & 21,0 & 2,4 & 53,1 & 14,2 & 100,0 & 100,0 & 100,0 \\
\hline Europe nordique & 2,1 & 18,3 & 3,1 & 58,9 & 14,6 & 109,2 & 124,6 & 120,2 \\
\hline $\begin{array}{l}\text { Europe } \\
\text { méditerranéenne }\end{array}$ & 0,4 & 15,5 & 1,3 & 40,5 & 10,9 & 62,4 & 75,5 & 67,1 \\
\hline $\begin{array}{l}\text { Europe centre- } \\
\text { orientale }\end{array}$ & 0,3 & 15,1 & 1,2 & 39,7 & $-7,3$ & 12,6 & 29,6 & 34,5 \\
\hline
\end{tabular}

Source : EUROSTAT.

Après l'effondrement des économies socialistes, une crise de transition profonde et l'explosion des inégalités sociales, les pays de l'Europe centre-orientale ont bénéficié d'une nette reprise au début des années 2000. Les économies s'y sont reconstruites sur la base d'une forte dépendance par rapport au capitalisme ouest-européen. Mais la crise de 2008 a signifié dans ces pays, sauf en Pologne, la fin des taux de croissance élevés qui permettaient d'espérer un rattrapage progressif du niveau moyen européen. Ils souffrent aussi aujourd'hui de la concurrence des pays émergents et d'une corruption importante, alors que leurs marchés intérieurs sont restreints par la faiblesse des salaires et une crise démographique (faibles fécondités, vieillissement de la population, émigration massive vers l'Europe occidentale, en particulier des jeunes bien éduqués). Les gouvernements d'Europe centre-orientale s'opposent globalement à une Europe plus sociale, puisque leur éventuel avantage comparatif réside, dans le cadre d'un marché unique, dans leurs bas salaires et leur faible protection sociale. Ils 
répugnent à une Europe plus verte pour les plus dépendants du charbon et de l'industrie lourde, au premier rang desquels se trouve la Pologne. a affecté l'ensemble de l'économie européenne, elle a plus durablement touché sa périphérie méridionale. Elle y a brisé la forte tendance au rattrapage des premières décennies de l'adhésion à l'Union européenne. Cette partie de l'Europe souffre d'un handicap technologique et de faibles niveaux de dépenses en recherchedéveloppement, alors que les salaires s'y sont peu à peu rapprochés de ceux de l'Europe du nord-ouest. Dans le cadre de la mondialisation, elle perd ainsi ses avantages comparatifs pour la production de faible ou moyenne technologie. L'introduction de l' $€$ l'empêche de compenser ces désavantages par une dépréciation monétaire et les pays «frugaux » du nord entendent contenir leurs déficits budgétaires et les transferts en sa faveur.

L'Europe nordique reste la plus redistributive. L'encadrement social y a été le mieux conservé. Le maintien de segments industriels nationaux de haute technologie garantit sa compétitivité. Les faibles densités de population, jointes à la rigueur morale, permettent à l'ensemble des forces socio-économiques de souscrire sans trop de réticences à des politiques environnementales résolues.

\section{La transcription du contexte global dans la composition du Parlement européen}

Ce contexte général de crise et d'atteintes aux transferts sociaux a généré un désenchantement envers la construction européenne promue par les partis de l'establishment, sociaux-démocrates, libéraux, démocrates-chrétiens et conservateurs europhiles. Un mécontentement diffus s'est développé parmi les couches sociales déclassées ou en risque de déclassement. Ces couches populaires estiment ne pas avoir de prise sur un establishment national et européen qui ne rencontre pas leurs préoccupations quotidiennes. La décomposition des structures d'encadrement traditionnelles du monde du travail a ainsi fait naître un nouveau clivage électoral, venu s'ajouter à ceux mis en évidence par Lipset et Rokkan (Lipset et Rokkan, 1967 ; Rokkan, 1970 ; Kriesi, 1998 ; Hooghe et Marks, 2018 : Gordon, 2018) : ce nouveau clivage se caractérise sur un de ses deux versants par le rejet des migrants, de la construction européenne, le repli national et différentes formes de populisme et de réflexes protectionnistes. Ce versant du clivage est occupé par les partis conservateurs eurosceptiques et la droite extrême (de sorte qu'il sera justifié dans la suite de cet article de totaliser leurs scores électoraux), mais aussi partiellement par des partis d'extrême-gauche. À l'opposé, parmi les gagnants de la nouvelle économie, des attitudes individualistes s'imposent, dans un contexte de compétitivité accrue et d'effacement des solidarités sociales. D'autres, plus altruistes, plutôt issus de groupes sociaux bien éduqués, prennent conscience de l'enjeu global des questions environnementales et sociales.

Quand et comment ces évolutions globales se sont-elles manifestées dans les comportements des électeurs? Le tableau 4 indique le pourcentage des parlementaires relevant des différents groupes politiques au Parlement européen depuis les premières élections au suffrage direct en 1979. Il ne peut être interprété qu'en termes de 
tendances générales, pour plusieurs raisons : les petits pays bénéficient d'une surreprésentation parlementaire ; certains partis ont glissé d'un groupe parlementaire à un autre au cours du temps ou ont modifié leurs positionnements ; le nombre de pays membres n'est pas resté constant, ce pourquoi nous avons distingué les six paysmembres originels, les autres membres occidentaux et ceux ayant adhéré suite à la chute du système soviétique. Nous avons aussi très grossièrement considéré que l'addition des parlementaires des groupes ECR, ID (ou de leurs prédécesseurs) et des non-inscrits représentait l'opposition europhobe de droite à l'establishment européen ; ceci est une approximation puisqu'on compte aussi quelques parlementaires d'extrême-gauche parmi les non-inscrits (mais ils sont en général eux aussi critiques envers les formes de la construction européenne). Cette vision évolutive utilise donc une classification partisane plus grossière que celle utilisée plus loin pour l'analyse fine des résultats des trois dernières élections ${ }^{4}$. Elle n'en fournit pas moins quelques conclusions intéressantes.

Les mutations post-fordistes n'ont pas affecté globalement les choix partisans de l'électorat européen jusqu'à la crise de 2008. À l'échelle de l'ensemble de l'Europe, les forces relatives en présence restent assez similaires en 2004 à ce qu'elles étaient en $1979: 76 \%$ des parlementaires sont issus des trois grandes familles traditionnelles en 2004, ils étaient $78 \%$ en 1979 ; les forces contestataires de droite sont stables également ( $13 \%$ au lieu de $10 \%$ ), de même que celles de gauche, extrême-gauche et écologistes : $12 \%$ pour les deux années. La seule modification à la gauche des partis d'establishment est l'effondrement des votes en faveur des partis d'extrême-gauche liés au mouvement communiste international historique (outre la social-démocratisation de l'ancien PCI), et, à l'inverse, la poussée des écologistes, toutefois modeste ( $6 \%$ des parlementaires en 2004). Ces constats restent identiques si on travaille à géométrie constante, dans le cadre des seuls six pays fondateurs de la construction européenne.

En revanche, les attitudes électorales évoluent fortement après la crise de 2008. Les parlementaires des trois grandes familles politiques traditionnelles passent de $76 \% \mathrm{du}$ total en 2004 à seulement $59 \%$ en 2019 , et même moins de la moitié dans les six pays originels. Parmi ces trois familles, seuls les libéraux se maintiennent; le recul est net pour les sociaux-démocrates et plus fort encore pour les démocrates-chrétiens et conservateurs classiques, qui rassemblaient encore $37 \%$ des députés en 2004 et seulement $24 \%$ en 2019 (de $34 \%$ à $19 \%$ dans les six pays fondateurs). 
Tableau 4 - Évolution de la composition du Parlement européen (1979-2019) (en \% du nombre total de parlementaires)

\begin{tabular}{|c|c|c|c|c|c|c|c|c|}
\hline \multirow{2}{*}{\multicolumn{2}{|c|}{$\begin{array}{l}\text { Répartition des } \\
\text { parlementaires }\end{array}$}} & \multirow{3}{*}{ 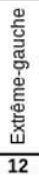 } & \multirow{3}{*}{ 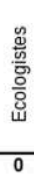 } & \multicolumn{4}{|c|}{ Partis centristes d'establishment } & \multirow{3}{*}{ 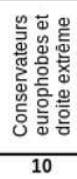 } \\
\hline & & & & \multirow{2}{*}{ बّٓ } & \multirow{2}{*}{ 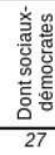 } & \multirow{2}{*}{ 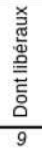 } & \multirow{2}{*}{ 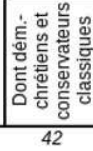 } & \\
\hline \multirow{9}{*}{ ฐّ̋ } & 1979 & & & & & & & \\
\hline & 1984 & 9 & 5 & 74 & 30 & 7 & 37 & 12 \\
\hline & 1989 & 8 & 8 & 74 & 35 & 9 & 30 & 9 \\
\hline & 1994 & 5 & 4 & 78 & 38 & 8 & 32 & 13 \\
\hline & 1999 & 7 & 8 & 74 & 29 & 8 & 37 & 12 \\
\hline & 2004 & 6 & 6 & 76 & 27 & 12 & 37 & 13 \\
\hline & 2009 & 5 & 7 & 72 & 25 & 11 & 36 & 15 \\
\hline & 2014 & 7 & 7 & 64 & 25 & 9 & 29 & 23 \\
\hline & 2019 & 5 & 10 & 59 & 21 & 14 & 24 & 26 \\
\hline \multirow{9}{*}{ 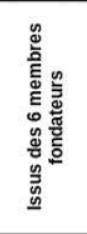 } & 1979 & 16 & 0 & 74 & 29 & 11 & 35 & 10 \\
\hline & 1984 & 12 & 5 & 69 & 28 & 9 & 32 & 14 \\
\hline & 1989 & 10 & 11 & 67 & 29 & 10 & 29 & 12 \\
\hline & 1994 & 4 & 6 & 74 & 31 & 7 & 35 & 16 \\
\hline & 1999 & 7 & 9 & 69 & 25 & 6 & 37 & 15 \\
\hline & 2004 & 6 & 9 & 75 & 27 & 13 & 34 & 10 \\
\hline & 2009 & 5 & 12 & 75 & 23 & 13 & 40 & 7 \\
\hline & 2014 & 6 & 8 & 63 & 27 & 8 & 28 & 23 \\
\hline & 2019 & 5 & 15 & 49 & 17 & 14 & 19 & 31 \\
\hline \multirow{9}{*}{ 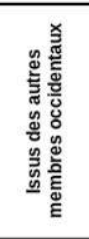 } & 1979 & 1 & 0 & 88 & 23 & 4 & 61 & 12 \\
\hline & 1984 & 4 & 3 & 85 & 35 & 2 & 49 & 8 \\
\hline & 1989 & 6 & 5 & 84 & 43 & 9 & 32 & 5 \\
\hline & 1994 & 7 & 1 & 84 & 48 & 8 & 28 & 7 \\
\hline & 1999 & 6 & 6 & 80 & 33 & 10 & 37 & 8 \\
\hline & 2004 & 6 & 5 & 79 & 32 & 10 & 36 & 10 \\
\hline & 2009 & 6 & 7 & 66 & 29 & 11 & 26 & 21 \\
\hline & 2014 & 12 & 7 & 56 & 27 & 9 & 20 & 25 \\
\hline & 2019 & 10 & 9 & 59 & 24 & 15 & 20 & 21 \\
\hline \multirow{4}{*}{ 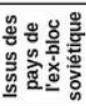 } & 2004 & 4 & 1 & 73 & 19 & 12 & 42 & 10 \\
\hline & 2009 & 3 & 1 & 76 & 24 & 10 & 43 & 21 \\
\hline & 2014 & 2 & 4 & 75 & 22 & 10 & 44 & 25 \\
\hline & 2019 & 1 & 3 & 73 & 21 & 15 & 37 & 21 \\
\hline
\end{tabular}

\section{Quatre macro-régions électorales, plus le Royaume- Uni}

Au-delà du rôle déterminant du contexte socio-économique général, on peut diviser l'Union européenne en quatre macro-régions, dans lesquelles on peut reconnaître, sans négliger les différences nationales, des similarités historiques, géographiques, de cultures politiques, de formes de coopération, de date d'accession à l'Union européenne et de comportements électoraux (tableau 5). La Grande-Bretagne à la veille du Brexit constitue un cas particulier: sa droite d'establishment a rompu avec la droite d'establishment européenne et sa social-démocratie est loin de partager unanimement les positions europhiles de la social-démocratie continentale. 
Tableau 5 - Résultats des élections européennes dans les 4 macro-régions de l'Union européenne (2009-2019)

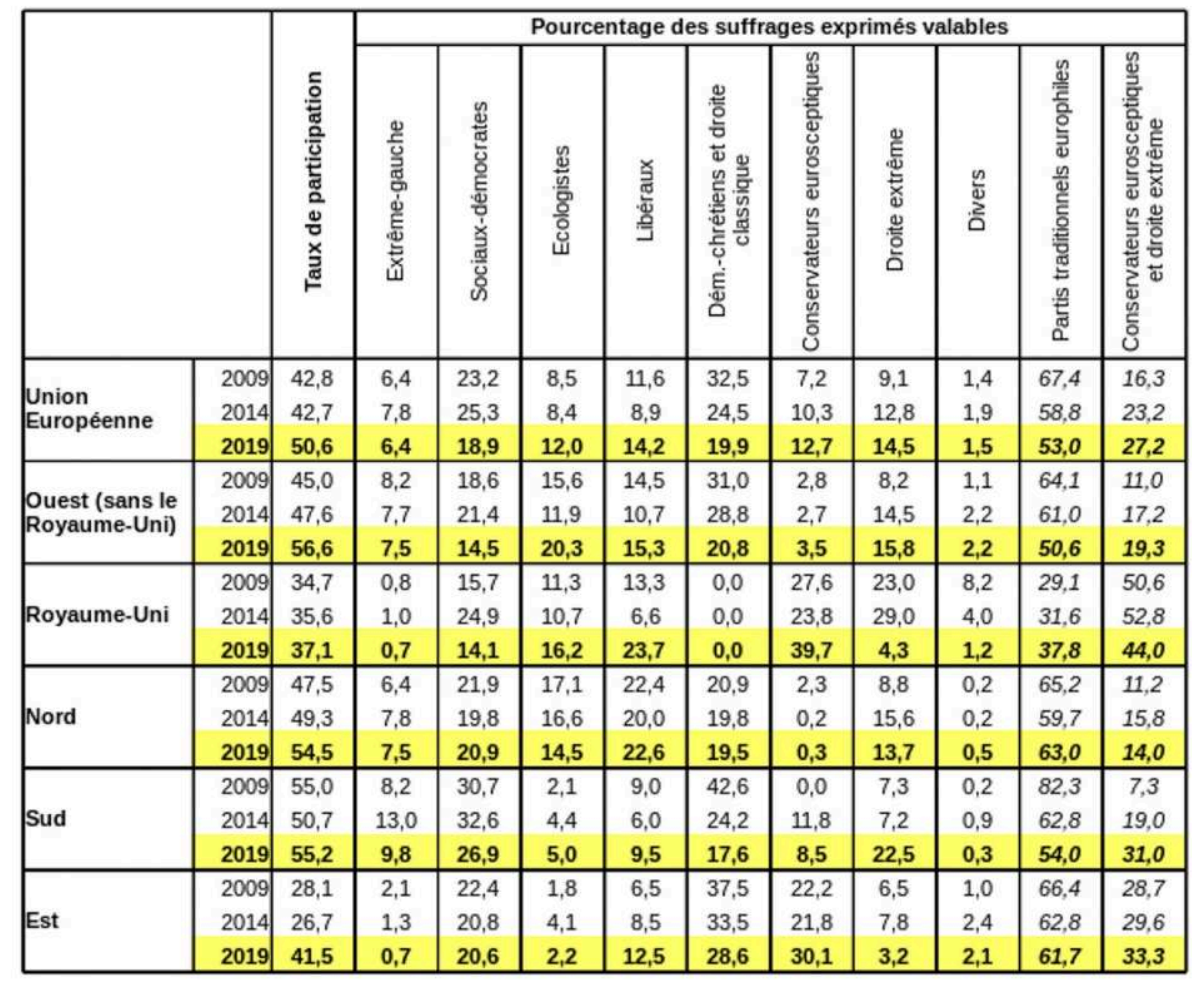

Ouest = Pays-Bas, Belgique, Luxembourg, France, Allemagne (y compris l'ex-RDA), Autriche, Irlande Nord = Danemark, Suède, Finlande.

Sud = Portugal, Espagne, Italie, Malte, Grèce, Chypre .

Est = Pays Baltes, Pologne, Tchéquie, Slovaquie, Hongrie, Slovénie, Croatie, Roumanie, Bulgarie. Les résultats nationaux sont pondérés par le nombre de suffrages exprimés valables; le taux de participation l'est par la population.

Les résultats de la Coalition européenne qui s'est présentée en Pologne en 2019 ont été ventilés à raison de 5/22 pour les sociaux-démocrates et de 17/22 pour les démocrates-chrétiens et la droite classique. Cette clé correspond à l'affiliation des députés de cette coalition aux groupes S\&D et PPE.

33 Le tableau 5 couvre les trois dernières élections européennes, période durant laquelle l'évolution des comportements politiques a été la plus marquée. Remarquons d'abord que, quels que puissent être les désenchantements de l'opinion publique envers l'Europe, les niveaux de participation n'ont pas faibli durant cette décennie, bien au contraire. Tout en restant les plus faibles, ils ont même très sensiblement progressé en Europe centre-orientale.

C'est en Europe nordique, où les évolutions socio-économiques ont été les moins clivantes, que les comportements politiques sont restés les plus stables. Néanmoins, la droite extrême y a progressé aux mêmes niveaux qu'en Europe du nord-ouest. À la différence de l'Europe du nord-ouest, les verts n'ont pas progressé, les préoccupations écologiques étant largement intégrées par les autres partis.

En Europe de l'ouest, en dehors de la situation atypique de la Grande-Bretagne, l'effondrement de la démocratie-chrétienne et de la droite classique représente la mutation majeure de la décennie, plus encore que la faiblesse social-démocrate. Cela reflète entre autres l'affaiblissement du clivage Église-État et d'un conservatisme s'appuyant sur des valeurs morales et religieuses traditionnelles, en particulier dans le monde rural. À l'inverse, les libéraux et surtout les verts progressent. Après une forte 
croissance entre 2009 et 2014, l'extrême-droite se stabilise autour de 15 \% des suffrages, près de $20 \%$ si on y ajoute des conservateurs eurosceptiques très à droite.

L'effondrement de la démocratie-chrétienne et de la droite classique est plus spectaculaire encore en Europe méditerranéenne. La droite extrême y en a été la principale bénéficiaire : elle réunit près d'un tiers des suffrages si on y ajoute les partis de droite eurosceptiques ${ }^{5}$. Les libéraux n'ont pas de forte tradition historique dans cette partie de l'Europe, qui était en retard de développement industriel lorsque le système partisan s'est cristallisé au XIX ${ }^{\mathrm{e}}$ et au début du $\mathrm{XX}^{\mathrm{e}}$ siècles. Un niveau de développement moindre qu'en Europe occidentale a aussi limité la croissance des écologistes. C'est en revanche dans cette partie de l'Europe que l'extrême-gauche réalise ses meilleures performances, à travers l'émergence de mouvements alternatifs comme Podemos en Espagne et SYRIZA en Grèce, dont les croissances ont toutefois été freinées après leur confrontation avec l'exercice du pouvoir.

En Europe centre-orientale, la droite se maintient à un niveau élevé, mais avec un net glissement interne vers le conservatisme eurosceptique aux dépens de la démocratiechrétienne europhile mise en place au lendemain de la chute du système soviétique avec l'appui affirmé des démocrates-chrétiens allemands. Les libéraux progressent, mais restent à des niveaux faibles. Les sociaux-démocrates, dont les appareils sont souvent issus de la reconversion des anciens partis communistes, se maintiennent autour de $20 \%$ des suffrages. L'extrême-gauche est quasi-inexistante.

\section{Les déterminants structurels des géographies électorales infranationales}

La surdétermination des contextes nationaux ne permet pas d'appréhender aisément l'impact potentiel des structures socio-économiques régionales à travers le seul examen global de cartes dressées à l'échelle européenne (fig. 1 à 9). Nous avons donc soumis les scores des familles politiques et le taux de participation dans les subdivisions territoriales ${ }^{6}$ à des analyses en composantes principales, pays par pays. Sur les axes déterminés par ces analyses ont été projetés des indicateurs socio-économiques disponibles à cette échelle à travers toute l'Europe. L'hypothèse est donc posée que ces indicateurs sont sous-jacents à la détermination des comportements électoraux (tableau 6). La prudence est évidemment de règle dans l'interprétation des résultats, pour éviter le piège des ecological fallacies. Les comparaisons entre pays sont ainsi normées et tentent d'effacer l'impact de la surdétermination des contextes nationaux. 
Tableau 6 - Variables contextuelles projetées sur les axes des analyses en composantes principales (ACP)

\begin{tabular}{|l|l|}
\hline \multicolumn{1}{|c|}{ Variables EUROSTAT } & \multicolumn{1}{|c|}{ Acronyme } \\
\hline Densité de population (2017) (valeur logarithmique) & DK \\
Évolution de la population (2010-2018) & CR.POP \\
Index global de compétitivité (2016)(a) & COMP \\
Pourcentage de plus de 65 ans (2017) & VIEUX \\
Pourcentage de 20-39 ans (2017) & JEUNE \\
Taux de chômage parmi les 15-74 ans (2018) & CHOM \\
PIB par hab. en équivalent de pouvoir d'achat (valeur logarithmique) & PROSP \\
Part de l'agriculture dans le PIB & I \\
Part de l'industrie, sans la construction, dans le PIB & II \\
Part de la construction dans le PIB & CON \\
Part du commerce, transport, hébergement et activités de restauration dans le PIB & COMM \\
Part de la logistique, des activités financières et de l'immobilier dans le PIB & IIIM1 \\
Part des activités spécialisées, scienti fiques et techniques et des services aux entreprises dans le PIB & IIIM2 \\
Part des activités administratives, de défense, d'éducation et de santé dans le PIB & IIINM \\
Taux d'emploi des 20-64 ans & TX.EMPL \\
\hline
\end{tabular}

(a) Index synthétique prenant en compte la stabilité macroéconomique et institutionnelle, les infrastructures, la santé, l'éducation de base, l'efficacité et la taille du marché, l'éducation supérieure et la formation continue, le niveau de l'innovation technologique et l'environnement entrepreneurial.

Une analyse supplémentaire des résultats britanniques (sans l'Irlande du nord) a été faite en ajoutant une variable binaire ECOSSE et une de la Belgique en ajoutant une binaire FLANDRE. 
Tableau 7 - Analyse en composantes principales (ACP)

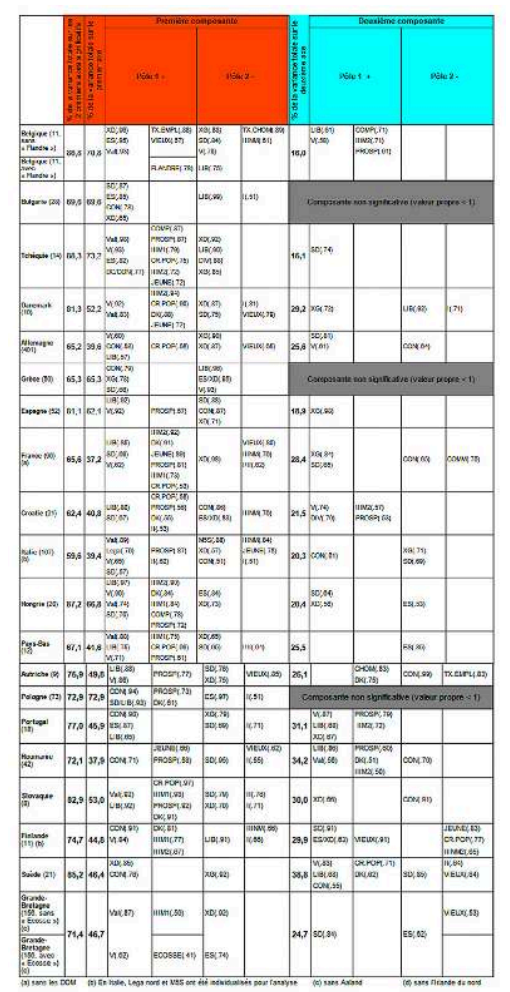

L'ACP est une analyse statistique multivariée, qui remplace les différentes dimensions caractérisant un nuage de points (ici les unités NUTS, caractérisées par les pourcentages qu'y obtiennent les différentes familles politiques) par un nouveau système d'axes, construits de manière telle qu'ils maximisent les proximités entre les observations. Les nouveaux axes (ou composantes) prennent successivement en compte la plus grande part de la variance totale. Les premiers axes reprennent donc la part la plus importante de l'ensemble de l'information : il est alors possible de se limiter à caractériser les points d'observation par leur position sur le ou les premiers axes, plutôt que par toute la série des pourcentages qu'y obtiennent les différents partis. On peut ensuite projeter sur ces axes un ensemble de variables indépendantes (ici des caractéristiques sociales, économiques ou démographiques) associées aux différents points d'observation, de manière à mettre en évidence les proximités que ces variables indépendantes entretiennent avec les configurations politiques mises en évidence.

Dans la première colonne, entre parenthèses, après le nom du pays, le nombre d'entités territoriales. La deuxième colonne indique la contribution des deux premiers axes de l'ACP à la variance totale. Les colonnes suivantes rendent compte de la variance portée par le premier et le deuxième axe, des familles politiques qui s'opposent significativement sur ceux-ci et de la projection sur ces axes des variables indépendantes. Les familles politiques et les variables indépendantes sont classées par ordre de corrélation (positive ou négative) décroissant avec les axes. Sont retenues dans le tableau les seules familles politiques et variables indépendantes corrélées significativement, au seuil de $5 \%$, et avec un taux de corrélation supérieur ou égal à 0,50.

39 Au-delà des niveaux de leurs scores nationaux, l'analyse permet ainsi de mettre en évidence les principales tendances structurantes des géographies des familles politiques. Elle peut déboucher sur des constats forts quant aux facteurs explicatifs sous-jacents. 
Tableau 8 - Coefficient de variation du vote pour les différentes familles politiques entre les entités électorales

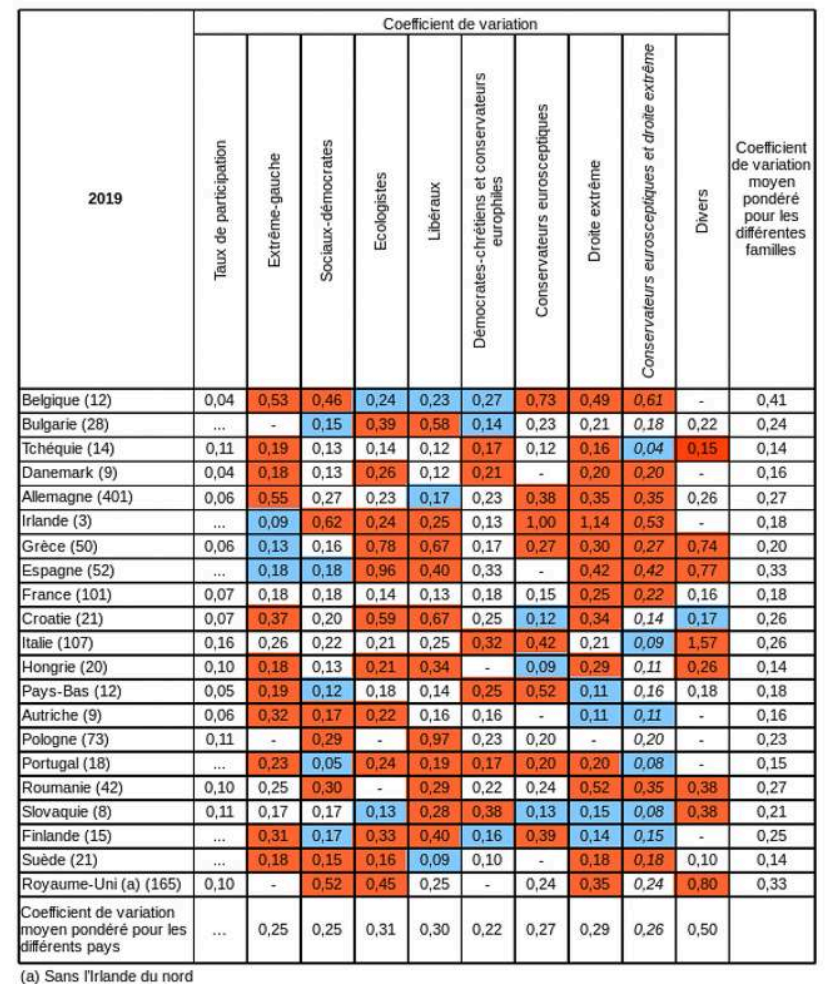

Sur fond rouge, les familles politiques dont le coefficient de variation est supérieur à la moyenne nationale de l'ensemble des familles. Sur fond bleu, celles dont le coefficient de variation est inférieur de plus de $75 \%$ à cette moyenne nationale.

\section{L'électorat démocrate-chrétien ou conservateur est le plus ubiquiste (illustration 5)}

En moyenne pondérée, sur l'ensemble des pays, le coefficient de variation des résultats des démocrates-chrétiens et conservateurs europhiles est le plus faible de toutes les familles politiques (tabl. 8). Cette ubiquité est néanmoins souvent teintée d'une tendance à des scores plus favorables dans les régions rurales ou semi-rurales et moins prospères, plus traditionalistes (tableau 9).

Des exceptions à un coefficient de variation inférieur à la moyenne nationale des familles politiques concernent toutefois six pays sur 20 :

- trois pays où la variabilité régionale des comportements électoraux est globalement faible pour l'ensemble des familles politiques, la Tchéquie, le Danemark et le Portugal ${ }^{7}$; - les Pays-Bas, où le CDA a des positions plus fortes dans les zones périphériques et dans le sud catholique ;

- la Slovaquie, où la forte variabilité spatiale des résultats démocrates-chrétiens reflète, outre la périphéricité de l'électorat des partis démocrates-chrétiens et conservateurs, l'appartenance au PPE des partis hégémoniques parmi la minorité hongroise dans le sud-ouest du pays ;

- enfin, l'Italie. Les succès de la Ligue, surtout dans le Nord, et de 5 Étoiles, surtout dans le Sud, ont brouillé la carte de l'ancienne démocratie-chrétienne (et de Forza Italia, qui en avait repris l'essentiel de l'électorat). Cette carte était assez uniforme, malgré une faiblesse dans le Centre-nord ${ }^{8}$. Les restes de Forza Italia et de ses petits alliés sont les 
plus forts dans le Sud, où la démocratie-chrétienne historique avait le caractère le plus clientéliste. Sur ces terres méridionales, 5 Étoiles prospère aujourd'hui, alors qu'au Nord et au Centre, le spectre politique s'est plus clairement recomposé autour d'une opposition entre la Ligue et la social-démocratie du PD.

La relative uniformité spatiale du vote démocrate-chrétien et conservateur classique pourrait s'expliquer par l'existence dans chaque pays d'un socle électoral interclassiste et donc d'un appui électoral dans différents types de configurations socio-spatiales.

Ajoutons à ces commentaires :

- en Allemagne, la force persistante du bastion social-chrétien bavarois et, à l'inverse, le maintien d'un déficit d'implantation de la CDU en Allemagne de l'est ;

- la situation particulière de la Pologne, où le contexte conservateur est tel que ce sont les partis rattachés au groupe PPE qui incarnent paradoxalement la modernité (les grandes villes et les zones à l'ouest de la frontière-fantôme de l'ancienne Allemagne), face au traditionalisme rigoriste du PiS (dans les anciens territoires russes et autrichiens, sauf leurs grandes villes);

- la Roumanie, où l'opposition entre la Transylvanie et les anciennes terres roumaines traduit à la fois la plus grande méfiance de la première envers la social-démocratie, considérée comme l'héritière de l'ancienne nomenklatura, et le poids qu'y ont les Hongrois, dont le parti ethnique est rattaché au PPE.

Le vote libéral est favorisé dans les régions prospères, compétitives, en croissance démographique, attirant les jeunes adultes. Ce contexte encourage aussi la participation électorale (illustrations 4 et 9)

dominante d'une corrélation positive entre vote libéral, densité de la population et
richesse des habitants, alors qu'une corrélation négative avec ces variables prévalait pour le vote démocrate-chrétien et conservateur. libéraux y sont issus d'une tradition agrarienne, avec des positions assez isolationnistes. C'est le cas de Venstre au Danemark; du parti du Centre en Suède (et partiellement du parti Libéral, qui tire une partie de son support d'une population rurale adhérant aux « Free churches " plutôt qu'à l'église officielle); du KESK finlandais (Vanlaer, 1984). En outre, les suédophones, très présents sur la côte Baltique de la Finlande, votent massivement pour le Parti suédois, affilié au groupe libéral. À l'inverse, dans ces pays luthériens, les partis de droite moderniste ne s'insèrent pas dans la tradition du clivage Église-État. Ils sont bien implantés dans l'électorat urbain. Ils sont affiliés au PPE (KF danois, Modérés suédois, KOK finlandais).

En Tchéquie, ANO21 pourrait tout autant être rattaché à la famille libérale qu'à la famille conservatrice eurosceptique, ce qui s'accorderait avec la géographie de son électorat: des scores plus élevés dans les régions de vieille industrialisation, comme le Nord-ouest, plus faibles à Prague. Enfin, en Bulgarie, les résultats libéraux sont gonflés par la présence de minorités turques, concentrées dans des zones moins prospères et représentées par le DPS, d'orientation libérale. 
Tableau 9 - Coefficients de corrélation entre les scores des écologistes, des libéraux, des démocrates-chrétiens et conservateurs europhiles et les variables « densités de population » et "PIB par habitant » (exprimées en logarithmes)

\begin{tabular}{|c|c|c|c|c|c|c|}
\hline \multirow{2}{*}{$\begin{array}{l}\text { Coefficients de } \\
\text { corrélation avec un } \\
\text { risque de non } \\
\text { s ignificativité } \\
\text { inférieur à } 10 \%\end{array}$} & \multicolumn{2}{|c|}{ Verts } & \multicolumn{2}{|c|}{ Libéraux } & \multicolumn{2}{|c|}{$\begin{array}{l}\text { Démocrates-chrétiens et } \\
\text { conservateurs europhiles }\end{array}$} \\
\hline & $\begin{array}{c}\text { Avec la } \\
\text { densité de } \\
\text { population }\end{array}$ & $\begin{array}{c}\text { Avec la } \\
\text { prospérité }\end{array}$ & $\begin{array}{c}\text { Avec la } \\
\text { densité de } \\
\text { population }\end{array}$ & $\begin{array}{c}\text { Avec la } \\
\text { prospérité }\end{array}$ & $\begin{array}{c}\text { Avec la } \\
\text { densité de } \\
\text { population } \\
\end{array}$ & $\begin{array}{c}\text { Avec la } \\
\text { prospérité }\end{array}$ \\
\hline Belgique (11) & $\mathrm{ns}$ & $\mathrm{ns}$ & ns & ns & $-0,594$ & ns \\
\hline Belgique FR (6) & ns & 0,907 & $n s$ & ns & ns & ns \\
\hline Belgique NL (5) & 0,849 & ns & ns & ns & $-0,971$ & ns \\
\hline Bulgarie (28) & - & - & $\mathrm{ns}$ & $-0,338$ & 0,367 & 0,351 \\
\hline Tchéquie (14) & 0,629 & 0,765 & $-0,524$ & $-0,798$ & 0,472 & 0,681 \\
\hline Danemark (10) & 0,868 & 0,541 & $\mathrm{~ns}$ & $\mathrm{~ns}$ & $\mathrm{~ns}$ & $\mathrm{~ns}$ \\
\hline Allemagne (401) & 0,593 & 0,487 & ns & 0,168 & $-0,385$ & $\mathrm{~ns}$ \\
\hline Grèce $(50)$ & ns & $\mathrm{ns}$ & $\mathrm{ns}$ & $\mathrm{ns}$ & $\mathrm{ns}$ & ns \\
\hline Espagne (52) & $\mathrm{ns}$ & 0,639 & $\mathrm{~ns}$ & 0,595 & $-0,356$ & $-0,518$ \\
\hline France (96) (a) & 0,426 & 0,548 & 0,510 & 0,678 & $\mathrm{~ns}$ & $\mathrm{~ns}$ \\
\hline Croatie (21) & 0,631 & $\mathrm{~ns}$ & $\mathrm{~ns}$ & $\mathrm{~ns}$ & $-0,567$ & $-0,623$ \\
\hline Italie (107) & $\mathrm{ns}$ & 0,654 & $\mathrm{~ns}$ & 0,379 & $\mathrm{~ns}$ & $-0,325$ \\
\hline Hongrie (20) & 0,632 & 0,626 & 0,894 & 0,783 & - & - \\
\hline Pays-Bas (12) & 0,511 & 0,763 & 0,695 & 0,674 & ns & $-0,525$ \\
\hline Autriche (9) & 0,691 & 0,858 & $\mathrm{~ns}$ & $\mathrm{~ns}$ & $-0,759$ & ns \\
\hline Pologne (73) & - & - & - & - & 0,441 & 0,563 \\
\hline Portugal (18) & 0,699 & 0,803 & 0,728 & 0,435 & ns & $-0,483$ \\
\hline Roumanie (42) & - & - & 0,641 & 0,750 & $-0,267$ & $\mathrm{~ns}$ \\
\hline Slovaquie (8) & - & - & 0,861 & 0,938 & ns & ns \\
\hline Finlande (11) (b) & 0,706 & 0,639 & $-0,596$ & $\mathrm{~ns}$ & 0,812 & ns \\
\hline Suède (21) & 0,370 & 0,521 & $\mathrm{~ns}$ & $\mathrm{~ns}$ & 0,805 & ns \\
\hline Angleterre (158)(c) & $\mathrm{ns}$ & 0,215 & $\mathrm{~ns}$ & 0,619 & $-0,657$ (d) & $-0,496$ (d) \\
\hline \multicolumn{7}{|l|}{ (a) sans les DOM } \\
\hline \multicolumn{7}{|l|}{ (b) sans Aaland } \\
\hline \multicolumn{7}{|c|}{ (c) Angleterre uniquement, sans le pays de Galles } \\
\hline
\end{tabular}

Les coefficients de corrélation significativement positifs au seuil de $10 \%$ sont en rouge, significativement négatifs en bleu. Entre parenthèses, le nombre d'entités territoriales.

Les contextes prospères, surtout métropolitains, favorisent aussi l'essor d'une gauche post-moderne écologiste en Europe du nord-ouest et du nord. Libéraux et écologistes y sont souvent en compétition ou en tout cas simultanément forts (illustration 3)

51 Les verts présentent, en moyenne pondérée, les variabilités infranationales les plus fortes (tabl. 9). Comme pour les libéraux, leur électorat est surtout localisé dans les aires métropolitaines et dans certaines zones périmétropolitaines les plus prospères, avec une population jeune, bien éduquée, une croissance démographique plus forte que la moyenne nationale, un poids important du tertiaire qualifié. Ceci est vrai dans tous les pays où le vote écologiste est significatif, en Europe occidentale et septentrionale.

52 Cela ne signifie pas qu'électeurs libéraux et écologistes soient interchangeables, encore que les transferts entre ces deux familles politiques ne soient pas négligeables. Il s'agit plutôt, au départ d'un recrutement privilégié dans une même base sociale, les classes moyennes aisées, jeunes et bien éduquées, d'un clivage entre ceux qui développent une sensibilité politique plus orientée vers la dérégulation et l'entrepreneuriat, et ceux qui privilégient l'écologie, le social et sont plus impliqués dans le non-marchand ${ }^{9}$.

53 En France, les verts (comme les libéraux et les sociaux-démocrates) sont globalement mieux représentés dans la moitié sud-ouest, à l'opposé de la plus forte présence de la droite extrême et eurosceptique dans la moitié nord-est (hors région parisienne), jadis la plus industrielle. Le fort vote écologiste en Corse peut traduire la volonté d'électeurs 
autonomistes de ne pas voter pour les autres partis, qui incarnent plus le centralisme de l'État.

En Espagne et en Grande-Bretagne, la carte écologiste est biaisée par le rattachement à cette famille d'une part importante des nationalistes catalans, basques et galiciens, d'une part, écossais et gallois de l'autre. En outre, les verts anglais sont moins métropolitains qu'ailleurs : ils sont aussi bien représentés dans les zones semi-rurales aisées du sud de l'Angleterre.

La désindustrialisation a estompé l'association entre industrie et bastions sociaux-démocrates et d'extrême-gauche, sauf là où une hystérésis s'est conservée à travers de puissantes structures d'encadrement. Une autre explication à cette déconnexion tient au fait que ce lien s'est plutôt mis en place lors des premières phases d'industrialisation que durant celle des Golden Sixties (illustration 2)

La géographie sociale-démocrate s'explique principalement, pays par pays, par des historicités, mais celles-ci ne peuvent toutes être rattachées à une tradition ouvrière. Il n'est plus possible d'établir, comme c'était encore le cas en Europe occidentale voici une soixantaine d'années, une corrélation entre le niveau de la social-démocratie et l'importance de l'industrie, en particulier l'extraction et l'industrie lourde. Selon les pays, les déterminants des points forts sociaux-démocrates sont divers, s'additionnent parfois, ce qui peut donner naissance à des tensions politiques entre visions contradictoires à l'intérieur des partis sociaux-démocrates. Le support peut provenir : - d'un électorat âgé, sociologiquement lié à l'ancienne industrialisation, parfois disparue aujourd'hui, dans des régions où les taux de chômage sont relativement élevés et le tertiaire non marchand surreprésenté par défaut, comme la Wallonie ou les anciens bassins industriels anglais. La condition en est la persistance des encadrements politiques, sociaux et culturels traditionnels ;

- d'un électorat de classes moyennes du secteur non marchand, dans les grandes villes (Paris, Bruxelles, Londres, Vienne), potentiellement concurrencé par les écologistes ;

- d'un électorat populaire issu de l'immigration, venant éventuellement renforcer dans les grandes villes l'électorat de classes moyennes ou l'électorat ouvrier traditionnel résiduel, voire s'y substituant ;

- des hystérésis d'opposition à la grande propriété foncière ou au métayage (sud de l'Espagne, Troisième Italie);

- de la superposition de l'anti-cléricalisme à la tradition ouvrière ou de luttes foncières (Wallonie vs. Flandre en Belgique ; Troisième Italie ; Allemagne occidentale protestante vs. catholique) ;

- en Europe centre-orientale, des héritages de réseaux clientélistes liés aux nomenklaturas, bien présents dans la vieille Roumanie rurale, Valachie et Moldavie, ou en Bulgarie.

57 En France, la concurrence de la droite extrême sur l'électorat populaire et d'origine ouvrière est telle que les anciens bassins industriels socialistes ont disparu de la carte. Les points forts socialistes apparaissent aujourd'hui assez similaires à ceux de l'électorat écologiste, à Paris et dans la moitié sud-ouest du pays, déconnectés d'une base ouvrière historique.

Bien plus faible que le vote social-démocrate, le vote d'extrême-gauche est très lié aux spécificités des histoires partisanes nationales et encore moins explicable par la géographie de l'ancienne industrialisation que le vote socialiste (illustration 1) 

géographie de l'extrême-gauche, en des lieux qui sont (ou étaient) simultanément des bastions sociaux-démocrates: ancien axe industriel wallon, Nord-Pas-de-Calais, Asturies. Mais pour le reste, les déterminants du vote d'extrême-gauche sont multiples : héritages des traditions de luttes agraires au sud du Portugal (alors que celles-ci avaient plutôt été encadrées par les socialistes dans le sud de l'Espagne); «ostalgie» dans les deux anciennes démocraties populaires qui étaient les plus industrielles et développées et possédaient avant-guerre des partis communistes importants, l'ex-RDA et dans une moindre mesure la Tchéquie; nouvelle gauche populiste à la géographie assez ubiquiste, métropolitaine mais pas seulement, en Espagne et en Grèce. Hors du Nord-Pas-de-Calais, la géographie de l'extrême-gauche française est devenue assez similaire à celle des socialistes et des écologistes (et sans doute, sur des bases sociales assez voisines, dans le non-marchand et une partie des classes moyennes en voie de prolétarisation) : Paris et la moitié sud-ouest de la France, la France " post-industrielle».

\section{Le vote protestataire de droite serait associé au déclin économique et} démographique (illustrations $6,7,8$ )

61 Ceci rendrait compte de la « revenge of the places that don't matter » (Rodriguez-Pose, 2018) et impose de considérer simultanément le vote eurosceptique conservateur et le vote de droite extrême. La frontière est parfois ténue entre les bases sociales, les électorats et les programmes de ces familles. La force respective de l'une ou l'autre selon les pays ne dépend dans bien des cas que des avatars de l'histoire de leurs systèmes partisans ${ }^{10}$.

62 Cette association n'est toutefois que partiellement vérifiée ${ }^{11}$. Certes, dans certains pays les foyers principaux de la droite extrême se retrouvent dans les zones les moins prospères et d'ancienne industrialisation (Hauts-de-France et Lorraine, ex-RDA en Allemagne, Nord-ouest et Nord-est de la Tchéquie). Dans ces cas on peut observer des similitudes entre cette géographie et celle de l'extrême-gauche, qui subsiste à titre plus ou moins résiduel dans les vieux bassins industriels. Mais là où les structures sociopolitiques d'encadrement de la classe ouvrière ont le mieux résisté (Allemagne occidentale, Wallonie, Angleterre), les zones d'ancienne industrialisation ne se sont pas transformées en bastions de la droite extrême (quelles que puissent être les attitudes sociologiques individuelles des électeurs de gauche traditionnels envers par exemple l'immigration).

63 Mais à côté d'anciennes zones de gauche passées à la droite extrême, on trouve, dans des cadres nationaux spécifiques, au moins deux autres configurations favorables à l'euroscepticisme conservateur et à la droite extrême :

- un conservatisme doublé d'attitudes anti-immigration, dans des zones plutôt non métropolitaines mais prospères (bien que parfois potentiellement menacées par une structure économique dominée par des petites et moyennes entreprises, moins aptes à s'intégrer dans la nouvelle économie globalisée dominée par la haute technologie), de villes moyennes ou de zones semi-rurales, de classes moyennes, souvent âgées, inquiètes pour la défense de leur statut et de leur sécurité supposée menacée (Vandermotten et al., 1990 ; Iammarino et al., 2019). C'est le cas par exemple en Flandre, en Angleterre, aux Pays-Bas, dans le Nord de l'Italie pour la Ligue, sur les décombres des structures d'encadrement de la démocratie-chrétienne consociative, voire dans le Centre dans d'anciens points forts du PCI, dont les structures coopératives se sont 
effondrées. En France méditerranéenne, le vote populaire d'anciens bastions d'extrême-gauche et le vote ultra-conservateur de populations âgées aisées se superposent pour favoriser l'extrême-droite, dans une région tôt confrontée à une immigration nord-africaine importante, alors que de nombreux Pieds-Noirs s'y étaient établis ;

- un conservatisme traditionaliste, nationaliste et religieux, populiste et interclassiste en Europe centre-orientale, moins présent dans les métropoles (PiS; FIDESz en Hongrie ; Slovaquie). Le vote pour l'UUP et le DUP en Irlande du nord peut être rattaché à la même catégorie.

Ajoutons le cas particulier du Mouvement 5 Étoiles, fort difficile à classer, au-delà de son euroscepticisme. Ses succès principalement dans le Sud italien, à l'inverse de la Ligue, montrent une géographie de région pauvre, mais où une partie importante de l'électorat a profité de l'effondrement des structures de la démocratie-chrétienne pour exprimer son refus du clientélisme, des compromissions et de la corruption. Il s'agit là d'un mélange entre volonté démocratique de réappropriation du contrôle de la chose publique et attitudes populistes de rejet de la classe politique jugée corrompue.

\section{Synthèse des principales oppositions spatiales}

Un retour à l'ACP permet une synthèse des principales oppositions spatiales au niveau infranational. Limitons-là à l'examen de la première composante, qui rend compte rappelons-le, selon les pays, de 37 à $73 \%$ de la variance totale.

Le contraste spatial le plus fréquent et le plus fort oppose les régions métropolitaines et riches aux régions périphériques, voire aux régions intermédiaires et à certaines portions du périurbain (tableau 10 et illustration 10). Ces régions centrales ont en général un vote plus écologiste, plus libéral, moins conservateur, moins d'extrêmedroite et des taux de participation plus élevés. On peut en inférer que cette transcription spatiale recouvre des oppositions sociales sous-jacentes (les gagnants de la mondialisation vs les autres).

67 Dans les régions riches non métropolitaines, dont la situation privilégiée peut apparaître menacée par la globalisation, le vote conservateur ou de droite extrême est conforté ou peut se développer (Nord, voire Centre, de l'Italie, Autriche), surtout quand il s'appuie sur des spécificités identitaires (Flandre en Belgique, Bavière en Allemagne).

Les zones de vieille industrialisation conservent très souvent des comportements électoraux spécifiques, soit que la gauche et/ou l'extrême-gauche y maintiennent des positions plus fortes (Wallonie, Ruhr, Asturies, Angleterre), soit qu'elles aient plutôt basculé vers l'extrême-droite et l'euroscepticisme (Tchéquie, Sud de l'ex-RDA, NordPas-Calais, Lorraine). 
Illustration 10 - Carte des scores sur la première composante des ACP, pays par pays

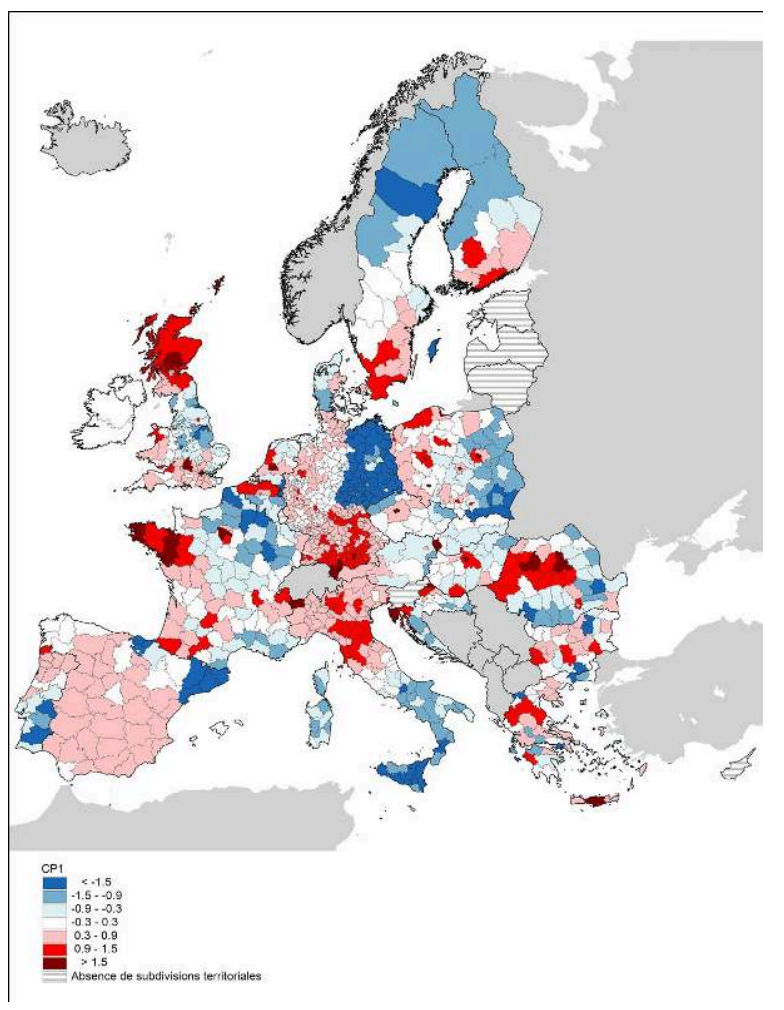


Tableau 10 - Synthèse des principales oppositions spatiales

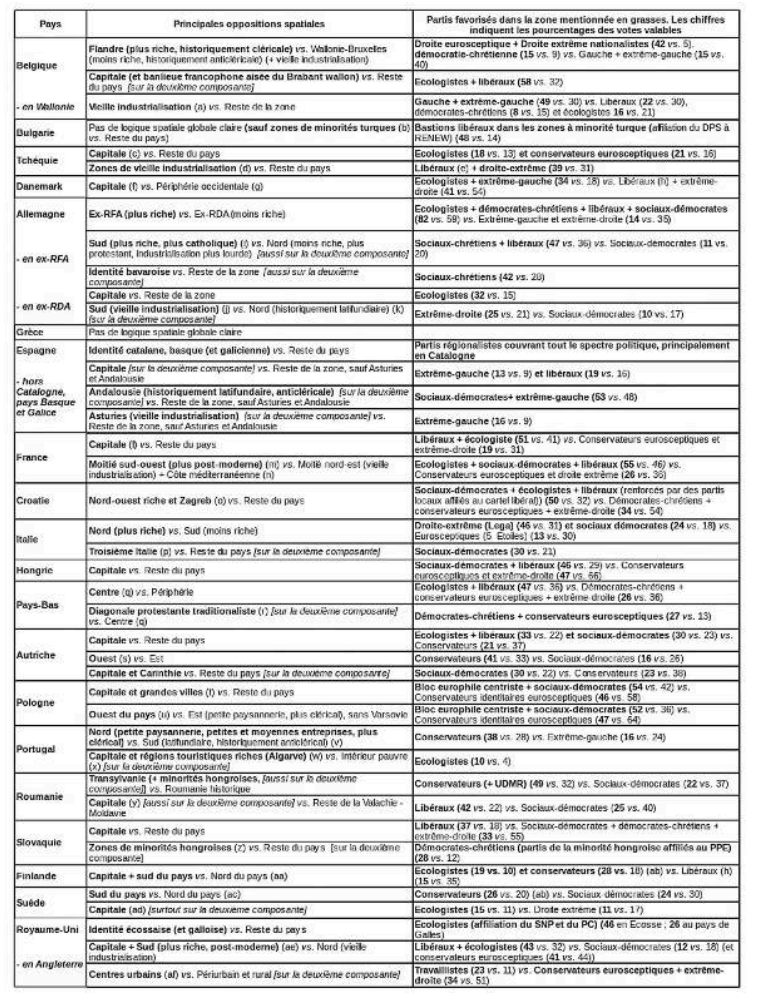

(a) Hainaut, Liège.

(b) Kardjali, Targovitse, Razgrad, Shumen, Silistra.

(c) Prague, Bohême centrale.

(d) Nord-Ouest, Liberec, Moravie centrale, Moravie-Silésie.

(e) Les libéraux tchèques, dirigés par «le Trump tchèque », le milliardaire Andrej Babiš, ont des positions populistes et eurosceptiques.

(f) Ville et banlieue de Copenhague.

(g) Nord, Ouest et Sud Jutland.

(h) Rappelons que les libéraux nordiques s'inscrivent en partie dans une tradition agrarienne.

(i) Bavière, Bade-Wurtemberg.

(j) Saxe, Saxe-Anhalt, Thuringe.

(k) Brandebourg, Mecklembourg - Poméranie antérieure.

(l) Ile-de-France

(m) Basse-Normandie, Bretagne, Pays de Loire, Grande-Aquitaine, Auvergne-Rhône-Alpes, Midi-

Pyrénées.

(n) Haute-Normandie, Hauts-de-France, Grand-Est, Centre, Bourgogne-Franche-Comté, Provence-Côte

d'Azur, Languedoc-Roussillon, Corse.

(o) Istrie, Primorje-Gorski (Rijeka), Medjumurje, Varaždin, Kropina-Zagorje, ville de Zagreb.

(p) Émilie-Romagne, Toscane, Marches, Ombrie.

(q) Hollande du nord, Utrecht.

(r) Zélande, Gueldre, Overijssel, Flevoland.

(s) Vorarlberg, Tyrol, Salzbourg.

(t) Entités NUTS 3 incluant les villes de plus de 200000 habitants (Varsovie, Cracovie, Lodz, Wroclaw, Poznan, Gdansk et Gdynia, Szczecin, Bydgoszcz et Torun, Lublin, Katowice, Bialystok, Czestochowa, Radom, Sosnowiec, Kielce).

(u) Warmskie, Pomorskie, Zachodnio-Pomorskie, Lubuskie, Dolnoslaskie, Opolskie, Slaskie, KujawskoPomorskie, Wielkopolskie.

(v) Au sud d'une ligne comprenant les districts de Lisbonne, Santarem et Castelo Branco.

(w) Districts de Lisbonne, Setubal, Faro.

(x) Districts de Bragance, Vila Real, Viseu, Guarda, Castelo Branco, Portalegre.

(y) Bucarest, llfov.

(z) Nitra, Trnava.

(aa) Circonscriptions électorales de Vaasa, Oulu, Laponie, Carélie du nord-Savonie du nord.

(ab) La base sociale et le positionnement des conservateurs nordiques est proche de celle des libéraux de l'Europe occidentale.

(ac) Norrland du centre et du nord.

(ad) Stockholm, Uppsala. 


\section{Conclusions}

On a montré précédemment que les variations spatiales des résultats aux élections européennes ne peuvent se ramener à quelques déterminants sociologiques simpl(ist)es, qui permettraient de transcender, dans une analyse unique, les frontières des pays européens (Vanlaer, 1984; Vandermotten et Medina Lockhart, 2002, 2015 ; Vandermotten et Vandeburie, 2011 ; Van Hamme, Vandermotten et Medina Lockhart, 2018). Néanmoins, cet article montre que, nonobstant la surdétermination des contextes nationaux, des déterminants à valeur générale peuvent être dégagés d'une analyse à l'échelle européenne.

Le contexte post-industriel, la globalisation, la poussée néo-libérale, la précarisation de larges pans de la population, la prolétarisation d'une partie des classes moyennes, ont entraîné une poussée de la droite extrême et de l'euroscepticisme, ainsi qu'une droitisation des positions des partis de l'establishment politique traditionnel. Ces derniers, qui ont été à la base de la construction européenne, s'affaiblissent: ils regroupaient $78 \%$ des parlementaires européens en 1979 et n'en rassemblent plus que $59 \%$ quarante ans plus tard. Cette tendance s'est accélérée après 2009. Elle affecte au premier chef les démocrates-chrétiens et les conservateurs europhiles, ainsi que les sociaux-démocrates. Les libéraux, souvent bien implantés parmi les classes moyennes post-modernes des régions prospères, s'en sortent mieux. Leurs bases électorales ont des similitudes avec celles des écologistes, qui représentent un autre versant, plus altruiste et socialement plus critique, issu du même groupe social. Globalement, la diversité des bases socio-géographiques des électorats des différentes familles politiques n'a fait que s'accentuer.

71 La critique du fonctionnement de la société contemporaine profite plutôt aux écologistes dans les régions gagnantes de l'Europe occidentale, aux droites extrêmes populistes voire radicales dans celles en déclin, sauf là où les encadrements par la gauche traditionnelle étaient les mieux organisés et ont résisté. Mais la droite extrême peut aussi être bien implantée dans des zones prospères, si elle peut s'y appuyer sur de puissantes hystérésis socio-politiques: la tradition conservatrice "blanche " en Angleterre, le nationalisme flamand, la tradition catholique confrontée à l'effondrement des structures d'encadrement de la démocratie-chrétienne en Italie du nord.

La crise a entraîné l'émergence d'une nouvelle extrême-gauche populaire (ou populiste ?) dans les pays méditerranéens, Espagne, Grèce, ou a conforté l'extrêmegauche traditionnelle là où celle-ci a su s'ouvrir à l'écologie politique, au Portugal. En revanche, en Italie, la social-démocratisation du PCI a entraîné son effondrement, en même temps que se dissolvaient les structures d'encadrement qui assuraient la consociativité et la cohésion sociale dans les bastions rouges.

73 En Europe centre-orientale, la conjonction du traditionalisme, du nationalisme, de l'anti-communisme et $\mathrm{du}$ désenchantement post-socialiste favorisent la droite conservatrice eurosceptique et extrême (en Pologne ou en Hongrie), quand ne se perpétuent pas des attitudes politiques marquées par le clientélisme, organisées par les nomenklaturas reconverties (en Roumanie ou en Bulgarie). Les déterminants électoraux se rapprochent plus de ceux de l'Europe occidentale en Tchéquie, en Slovénie et dans les pays Baltes. 
Le croisement des différentes observations montre que la théorie des clivages (Lipset et Rokkan, 1967; Rokkan, 1970) reste intéressante pour comprendre les géographies électorales dans les différents pays européens, à condition de l'utiliser de manière différenciée en fonction des histoires politiques nationales, de la réinterpréter et de ne pas amalgamer abusivement clivages sociaux et contrastes géographiques :

- le clivage Capital / Travail reste essentiel, mais les partis de gauche classique et d'extrême-gauche sont de moins en moins hégémoniques sur le versant Travail (s'ils l'ont jamais été là où ils étaient concurrencés par une démocratie-chrétienne puissante). Une partie de l'électorat « indigène » lié au monde du travail a glissé vers des partis de droite conservatrice eurosceptique ou extrême ;

- le clivage Église / État s'estompe sous l'effet de la déchristianisation, entraînant le recul des partis démocrates-chrétiens et conservateurs europhiles (sauf en Pologne et en Hongrie, où on assiste à une reconquête de l'État par un christianisme réactionnaire agressif, et en Bavière, où la CSU exprime plutôt un conservatisme identitaire en zone prospère) ;

- les clivages Centre / Périphérie et Urbain / Rural se sont transformés en un clivage Régions gagnantes de la globalisation / Régions en déclin. Il reprend certaines composantes des clivages traditionnels Capital / Travail et État / Église. Il est souvent devenu le clivage premier, en tout cas si on se limite à son expression géographique, sans analyser ses déterminants sous-jacents ;

- un nouveau clivage Économie (Moderne) / Écologie (Post-moderne) s'est constitué, qui distancie les motivations d'une partie de l'électorat de comportements liés aux clivages Capital / Travail et Église / État. Il recoupe en partie le clivage précédent.

Enfin, là où des partis puissants s'appuient sur de fortes identités régionales (Écosse, pays de Galles, Flandre, Catalogne, pays Basque, Sud-Tyrol, voire Bavière), on ne peut, comme le faisait Rokkan, ramener les succès de ces partis, dans des régions généralement prospères, à un simple positionnement périphérique sur un axe Centre / Périphérie, même quand ces régions s'opposent frontalement au gouvernement central.

\section{BIBLIOGRAPHIE}

Bussi M., Vandermotten C., Van Hamme G, 2012. Les dimensions spatiales de la droitisation de la classe ouvrière. In De Waele J.M., Vieira M. (ed.), Une droitisation de la classe ouvrière en Europe. Paris, Economica. p. 69-93

Caramani D, 1994. La nationalisation du vote :étude comparative des processus d'homogénéisation territoriale du soutien électoral des partis politiques dans 15 pays européens. Genève, Université de Genève.

Clark T.N., Lipset S.M. (ed.), 2001. The Breakdown of Class Politics. A Debate on Post-industrial Stratification. Washington DC, Woodrow Wilson Center Press.

Deutsch K.W., Foltz W.J. (ed.), 1966. Nation-Building. Routledge. 
Dijkstra L., Poelman H., Rodriguez-Pose A., 2019. The geography of EU discontent. Regional Studies [En ligne], vol. 54, n 6, p.737-753. DOI: https://doi.org/10.1080/00343404.2019.1654603

Dogan M., 1996. Classe, religion, parti : Triple déclin dans les clivages électoraux en Europe. Revue internationale de politique comparée, $\mathrm{n}^{\circ}$ 3, p. 515-540.

Evans G. (ed.), 1999. The End of Class Politics ? Class Voting in Comparative Context. Oxford, Oxford University Press.

EVS, 2008. EVS Variable Reports. Tilburg University and GESIS Leibniz Institute for the Social Sciences.

Flora P. (ed.), 1999. State Formation, Nation-building and Mass politics in Europe. The theory of Stein Rokkan based on his collective works. Oxford, Oxford University Press.

Franklin M., Mackie T., Valen H. (ed.), 1992. Electoral Change: Response to Evolving Social and Attitudinal Structure in Western Countries. Cambridge, Cambridge University Press.

Gellner E., 1983. Nations and Nationalism. Ithaca, Cornell University Press.

Gordon I.R., 2018. In what sense left behind by globalisation? Looking for a less reductionist geography of the populist surge in Europe. Cambridge Journal of Regions. Economy and Society [En ligne], vol. 11, n 1, p. 95-113. DOI: https://doi.org/10.1093/cjres/rsx028

Hemerijck A., 2013. Changing welfare states. Oxford, Oxford University Press.

Hooghe L., Marks G., 2018. Cleavage theory meets Europe's crises: Lipset, Rokkan, and the transnational cleavage. Journal of European Public Policy [En ligne], vol. 25, n 1, p. 109-135. DOI: https://doi.org/10.1080/13501763.2017.1310279

Ignazi P., 1999. Les partis d'extrême-droite : les fruits inachevés de la société postindustrielle. In Perrineau P. (éd.), Les Croisés de la société fermée. Paris, Cevipof. p. 126-142.

Iammarino S., Rodriguez-Pose A., Storper M., 2019. Regional inequality in Europe: Evidence, theory and policy implications. Journal of Economic Geography [En ligne], vol. 19, n 2, p. 273-298. DOI: https://doi.org/10.1093/jeg/lby021

Kitschelt H., 1994. The Transformation of the European Social Democracy. Cambridge, Cambridge University Press.

Kriesi H., 1998. The Transformation of Cleavage Politics. The 1997 Stein Rokkan Lecture. European Journal of Political Research, $\mathrm{n}^{\circ} 33, \mathrm{p}$. 165-185.

Levy J., 2013. Réinventer la France. Paris, Fayard.

Levy J., 2017. Atlas politique de la France. Les révolutions silencieuses de la société française. Paris, Autrement.

Lipset S.M., Rokkan S. (ed.), 1967. Party Systems and Voters Alignment : Cross-National Perspectives. Toronto, The Free Press.

Nordsieck W., 2019 (3d. ed.). Parties and Elections in Europe. Parlementary Elections and Governments since 1945. European Parliament Elections. Political Orientation and History of Parties. Deutsche Nationalbibliothek.

Rodriguez-Pose A., 2018. The revenge of the places that don't matter (and what to do about it). Cambridge Journal of Regions. Economy and Society [En ligne], vol. 11, n 1, p. 189-209. DOI: https:// doi.org/10.1093/cjres/rsx024

Rokkan S., 1970. Citizens, Elections, Parties. Oslo, Universitetforlaget. 
Trechsel A.H., Mair P., 2009. When parties (also) position themselves : an introduction to the EU profiler. EUI Working Paper, RSCAS 65, Florence, European Institute, European Union Democracy observatory.

Van Der Eijk C., Franklin M.F., 1996. Choosing Europe? The European Electorate and National Politics in the Face of Union. Ann Arbor, The University of Michigan Press.

Vandermotten C., Capron H., Decroly J.M., Romus P., 1990. Les régions et l'Europe. In Actes du gème Congrès des Économistes belges de langue française. Namur, CIFoP.

Vandermotten C., Medina Lockhart P., 2002. Electoral Geography Of Europe. In Perrineau P., Grunberg G., Ysmal C. (ed.), Europe at the Polls: The European Elections of 1999. New York, Palgrave, p. 181-206.

Vandermotten C., Medina Lockhart P., 2015. Les élections européennes de 2014 : une analyse comparative des résultats aux échelles nationale et régionale. EchoGéo [En ligne], Sur le Vif. DOI: https://doi.org/10.4000/echogeo.14303

Vandermotten C., Vandeburie J., 2011 (2 éd.). Territorialités et Politique. Bruxelles, Éditions de l'Université de Bruxelles.

Van Hamme G., 2012. Social Classes and Political Behaviours : Directions for a Geographical Analysis. Geoforum, vol. 43, n 4, p. 772-783.

Van Hamme G., Vandermotten C., Medina Lockhart P., 2018. The Electoral Geography of the Left in Western Europe since 1945: Permanences and Changes. Tijdschrift voor Economische en Sociale Geografie [En ligne], vol. 109, n 2, p. 274-294. DOI: https://doi.org/10.1111/tesg.12280

Vanlaer J., 1984. 200 millions de voix. Une géographie des familles politiques européennes. Bruxelles, Société royale belge de géographie.

Zarycki T., 2015. The electoral geography of Poland : Between Stable Spatial Structures and their Changing Interpretations. Erdkunde, vol. 69 n² 2, p. 107-124.

\section{ANNEXES}

\section{Composition des familles politiques}

Le tableau est limité aux partis ayant obtenu au moins $5 \%$ des voix ou un député au PE à l'une des élections de 2009, 2014 ou 2019. 

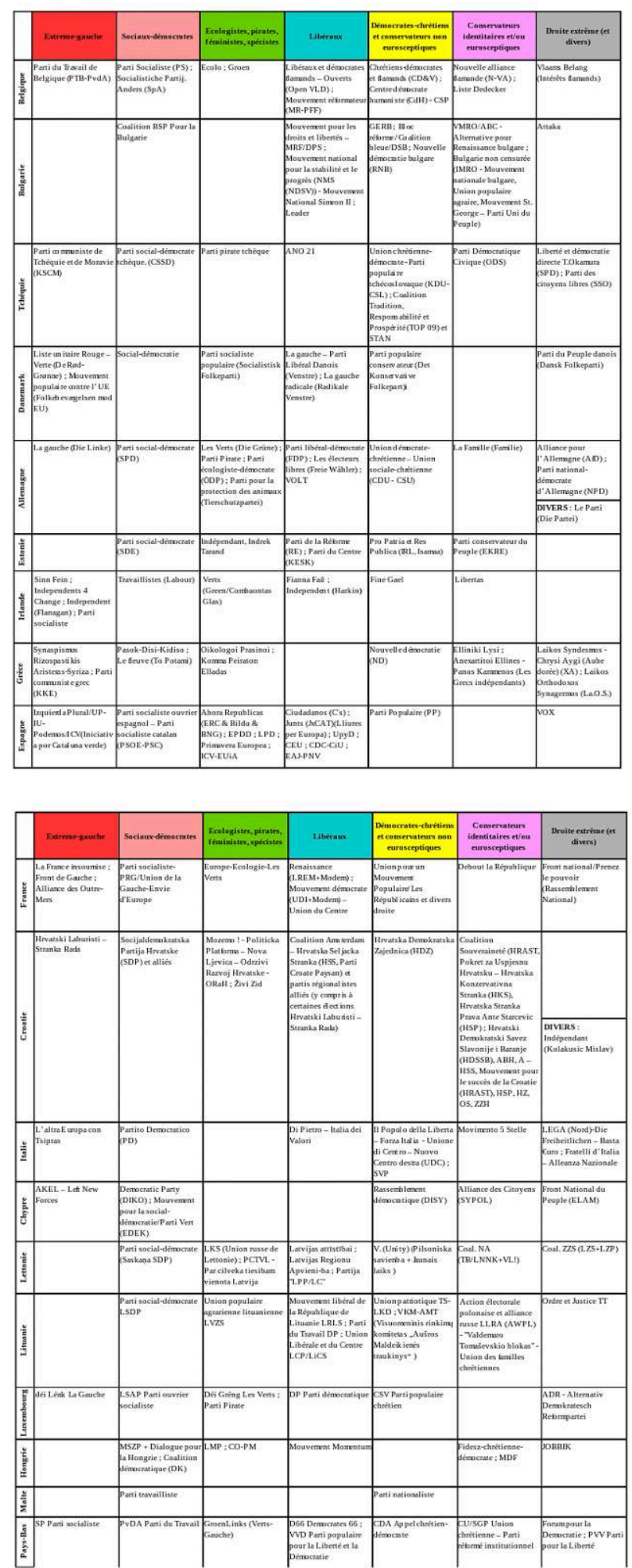


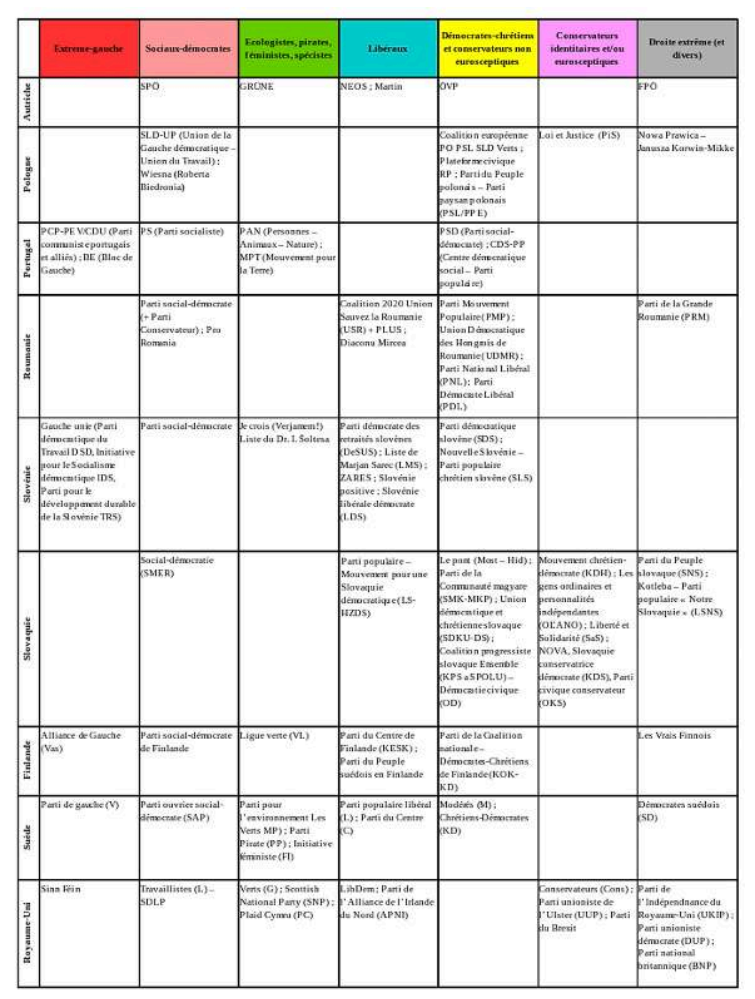

\section{Notes du tableau en Annexe :}

- Belgique : en Belgique, les partis d'une même famille politique relevant de chaque aile linguistique sont indépendants les uns des autres, sauf le PTB-PvdA (extrême-gauche), resté unitaire.

- Bulgarie : le MRF/DPS (Mouvement pour les Droits et les Libertés) représente la minorité turque.

- Tchéquie : le Parti des Citoyens libres (SSO), rattaché au groupe libéral au PE jusque 2017, occupe une position intermédiaire entre la droite eurosceptique et la droite extrême populiste. De même, ANO21, bien que faisant partie depuis 2014 du groupe ADLE au PE, de sorte que nous l'avons rattaché à la famille libérale, a des positions plutôt eurosceptiques. Il développe un discours populiste qui le rapproche des conservateurs eurosceptiques.

- Danemark : le Mouvement du Peuple contre l'UE est un parti eurosceptique de gauche. Il est affilié au groupe GUE/NGL depuis 2017. Le Parti socialiste populaire est un parti rouge-vert, affilié au groupe GUE/GVN jusque 2004.

- Allemagne : Tierschutz, parti de défense des animaux, a été rattaché à la famille écologiste, même s'il fait partie du groupe GUE/GVN au PE. VOLT a été rattaché à la famille libérale. Die Partei, parti satirique, apparaît inclassable.

- Irlande : le Sinn Fein, qui prône des options socialistes, a été rattaché à l'extrêmegauche du fait de son appartenance au groupe GUE/GVN au PE. Le Fianna Fail a été placé dans la famille libérale, du fait de son appartenance au groupe Renew au PE, et aussi suite à l'évolution récente de ses positions politiques, même si cela peut apparaître étonnant dans une perspective historique qui le rattacherait plutôt à la tendance conservatrice. 
- Espagne : le classement des partis est rendu assez difficile par les cartels électoraux dans lesquels s'inscrivent les partis régionalistes et qui ne correspondent parfois que partiellement à leurs positionnements politiques. Ainsi on pourrait rapprocher le BNG (Bloc national galicien) de l'extrême-gauche, l'ERC catalan de la social-démocratie ou le PNV (Parti nationaliste basque) de la démocratie-chrétienne.

- Croatie : Živi zid a été considéré comme un parti eurosceptique de gauche, avec des sensibilités environnementales, s'apparentant à un parti pirate et rattaché à ce titre à la famille écologiste. Le classement des partis croates entre libéraux, démocrateschrétiens et conservateurs europhiles et eurosceptiques est rendu assez incertain par l'effet des cartels électoraux, outre le rattachement à ceux-ci de partis régionalistes.

- Italie : clairement eurosceptique, le Mouvement 5 Étoiles est loin d'avoir toutes les caractéristiques d'un parti conservateur.

- Lettonie : la coalition LZS-LZP a évolué vers un euroscepticisme de droite populiste au départ de formations initialement agrariennes (LZS) et écologistes (LZP).

- Lituanie : LVZS a été classé parmi les écologistes, auxquels il se rattache au PE. S'il était initialement un parti agrarien eurosceptique, il a évolué, avec ses alliés, vers un positionnement vert et paysan modernisé.

- Pays-Bas : CU/SGP a été rattaché au groupe des conservateurs eurosceptiques, bien qu'un de ses deux députés au PE soit membre du groupe PPE. Forum pour la Démocratie $(\mathrm{FvD})$ a été classé dans la droite extrême, bien que ses députés fassent partie du groupe ECR : ce parti conservateur est divisé entre une aile plus axée sur l'économie et une autre, actuellement majoritaire, plus identitaire. Le parti a attiré tant des électeurs du VVD et du CDA que d'autres du PVV de Wilders.

- Pologne : la grande coalition centriste europhile opposée au PiS s'est répartie au PE entre 17 députés qui ont rejoint le PPE et 5 qui se sont affiliés au groupe S\&D.

- Roumanie : le Parti social-démocrate, dont Pro Romania est une dissidence sur un programme anti-corruption, s'est allié au petit Parti conservateur, dont on ne peut donc isoler les résultats. USR s'est aussi constitué sur une base anti-corruption. Le PNL (et sa dissidence du PDL) est le parti libéral historique, mais il a rejoint le groupe PPE en 2014.

- Suède : les Démocrates suédois ont été considérés comme un parti de droite extrême, bien qu'ils soient affiliés au groupe ECR.

- Royaume-Uni : le SNP et Plaid Cymru ont été rattachés aux écologistes sur le base de leur affiliation au PE. Ce sont évidemment avant tout des partis régionalistes, même s'ils ont des positions sociales-démocrates et favorables à une politique environnementale active.

\section{NOTES}

1. La variable utilisée est le pourcentage des suffrages exprimés valables (soit hors blancs et nuls) par rapport au nombre d'électeurs potentiels.

2. Les niveaux NUTS sont les niveaux hiérarchiques de découpage utilisés par EUROSTAT pour les statistiques régionales européennes. La plupart des pays sont traités jusqu'au niveau NUTS 3 (soit 
le département en France). Pour le Portugal, la Finlande et le Royaume-Uni (à l'exception de l'Irlande du Nord, non subdivisée), les données disponibles au niveau NUTS 3 pour les variables contextuelles ont été ajustées aux limites des circonscriptions électorales de taille similaire. La Belgique, l'Irlande, les Pays-Bas et l'Autriche ne sont traités qu'au niveau NUTS 2. L'Estonie, Chypre, la Lettonie, la Lituanie, le Luxembourg, Malte et la Slovénie ne sont pas subdivisés.

3. Nous ne parlons pas ici de la crise de la COVID, puisqu'elle est survenue après les élections européennes.

4. Ainsi, les conservateurs britanniques ont été classés avec les démocrates-chrétiens et conservateurs classiques tant qu'ils faisaient partie du groupe DE ou PPE, mais ils passent dans le groupe des conservateurs europhobes ensuite ; le FIDESz hongrois reste toujours classé parmi les démocrates-chrétiens et conservateurs classiques, ce qui ne s'accorde certainement plus à ses positions politiques actuelles.

5. Parmi lesquels le Mouvement 5 Étoiles a été inclus : bien que très hétérogène et difficilement classable, son actuelle débandade montre qu'une part importante de son électorat et de ses cadres tend à rejoindre la Ligue.

6. Du moins dans les pays subdivisés en un nombre suffisant d'entités, à savoir l'Allemagne, l'Autriche, la Belgique, la Bulgarie, la Croatie, le Danemark, la Tchéquie, l'Espagne, la Finlande, la France, la Grèce, l'Italie, la Hongrie, les Pays-Bas, la Pologne, le Portugal, la Roumanie, le Royaume-Uni, dont l'Angleterre, la Slovaquie, la Suède.

7. Même si dans le cas portugais l'opposition traditionnelle entre la force de la droite dans le Nord minifundiaire et de l'extrême-gauche dans le Sud latifundiaire se lit toujours sur la carte, en particulier pour les scores de l'extrême-gauche.

8. Il en va de même en France où une grande part de l'électorat conservateur s'est reporté sur La République en Marche, avec pour conséquence l'ubiquité de la carte de la famille libérale.

9. Alors que libéraux et écologistes sont tous deux bien représentés à Paris, cette dichotomie pourrait expliquer une plus forte présence libérale dans sa périphérie occidentale, une plus forte présence écologiste au sud de la capitale. À un niveau plus fin que celui de cette étude, celui des communes, on peut observer les mêmes oppositions à l'intérieur des communes aisées de Bruxelles et de sa périphérie aisée francophone du Brabant wallon.

10. S'il y a un lien entre croissance de l'euroscepticisme et du vote en faveur de partis populistes de droite ou de droite extrême, les deux discours se nourrissant mutuellement, les motivations de ce vote dans le chef des électeurs ne relèvent pas nécessairement de l'euroscepticisme. Dijkstra, Poelman et Rodriguez-Pose (2019) montrent ainsi que le mécontentement envers les formes prises par la construction européenne est, d'après les enquêtes d'opinion, faible au Danemark, alors que les partis eurosceptiques y font des scores significatifs, mais l'inverse prévaut en Espagne. Les programmes de ces partis rejoignent plus étroitement les sensibilités de leur électorat sur le rejet de l'immigration, même là où celle-ci est d'ampleur très limitée, ce qui renvoie donc à un enjeu identitaire symbolique.

11. Au-delà du fait qu'il convient de se garder de l'ecological fallacy que constituerait la confusion entre région en déclin et sentiment individuel de déclassement économique, social et/ou culturel de l'électeur. Ce ne sont pas nécessairement les électeurs les plus défavorisés qui votent le plus à l'extrême-droite dans les régions en déclin. 


\section{RÉSUMÉS}

Les élections européennes permettent de dresser une géographie électorale simultanée, qui pose toutefois des problèmes méthodologiques, dont celui de la définition de familles politiques. Les résultats restent très déterminés par les cadres étatiques. L'élection de 2019 se situe dans un contexte de crise systémique. La position dominante des partis démocrates-chrétiens et conservateurs et des sociaux-démocrates s'est fortement affaiblie depuis 2009. En revanche, les écologistes et les libéraux progressent, mais plus encore les partis eurosceptiques et de droiteextrême. Le contraste géographique principal oppose les régions métropolitaines gagnantes de la mondialisation au reste des territoires, surtout les zones en déclin économique et démographique. Mais l'euroscepticisme et la droite extrême se développent aussi dans des régions prospères non métropolitaines, qui pourraient être menacées par les conséquences de la globalisation et/ou qui nourrissent des sentiments identitaires.

The European elections allow to draw up a simultaneous electoral geography, which however poses methodological problems, among which the need to define political families. The results remain very determined by national frames. The 2019 election takes place in a context of systemic crisis. The dominant position of the christian-democratic, conservative and socialdemocratic parties has been sharply reduced since 2009. In contrast, greens and liberals are making progress, but the strongest progression is that of the eurosceptic and far-right parties. The main geographical contrast opposes the metropolitan areas to the rest of the territories, mainly the economic and demographic shrinking regions. But euroscepticism and the far-right are also growing in prosperous non-metropolitan regions, which could be threatened by the consequences of globalization and /or which nourish feelings of identity.

\section{INDEX}

Mots-clés : Europe, élection européenne, géographie électorale, crise systémique

Keywords : Europe, European election, electoral geography, systemic crisis

Thèmes : Sur le Champ - Sur le Terrain

\section{AUTEURS}

\section{CHRISTIAN VANDERMOTTEN}

Christian Vandermotten, cvdmotte@ulb.ac.be, est Directeur de la Classe des Lettres de l'Académie royale de Belgique et professeur émérite à l'Université libre de Bruxelles.

\section{PABLO MEDINA LOCKHART}

Pablo Medina Lockhart, pmedinal@ulb.ac.be, est chargé de recherches à l'Université libre de Bruxelles.

\section{HERMAN VAN DER WUSTEN}

Herman van der Wusten, h.h.wusten@contact.uva.nl, est Professeur émérite à l'Université d'Amsterdam. 